\title{
THE GENETICS OF BREAST AND OVARIAN CANCER IV: A MODEL OF BREAST CANCER PROGRESSION
}

\author{
By Baopeng Lu, Angus Macdonald and Howard Waters
}

\begin{abstract}
Gui et al. (2006), in Part III of a series of papers, proposed a dynamic family history model of breast cancer (BC) and ovarian cancer (OC) in which the development of a family history was represented explicitly as a transition between states, and then applied this model to life insurance and critical illness insurance. In this study, we extend the model to income protection insurance (IPI). In this paper, Part IV of the series, we construct and parameterise a semi-Markov model for the life history of a woman with BC, in which events such as diagnosis, treatment, recovery and recurrence are incorporated. In Part V, we then show: (a) estimates of premium ratings depending on genotype or family history; and (b) the impact of adverse selection under various moratoria on the use of genetic information.
\end{abstract}

\section{KEYWORDS}

Breast Cancer; BRCA1 Gene; BRCA2 Gene; Family History; Income Protection Insurance

\section{CONTACT ADDRESS}

Angus Macdonald, Department of Actuarial Mathematics and Statistics and the Maxwell Institute for Mathematical Sciences, Heriot-Watt University, Edinburgh EH14 4AS, U.K. Tel: +44(0)131-451-3209; Fax: +44(0)131-451-3249; E-mail: A.S.Macdonald@ma.hw.ac.uk

\section{INTRODUCTION}

\subsection{Income Protection Insurance and Genetics}

Income Protection Insurance (IPI), previously known as permanent health insurance (PHI), is designed to cover a proportion of the policyholder's salary should illness or disability prevent them from working.

Breast cancer $(\mathrm{BC})$ is a common and severe disease. Hereditary forms account for a small proportion of all cases, partly due to highly penetrant and dominant mutations in the BRCA1 and BRCA2 genes. Women with these mutations are very likely to develop $\mathrm{BC}$ and to develop it at relatively young ages. Today, women considered to be at high risk of $\mathrm{BC}$ can be offered a presymptomatic genetic test. This has led to debates about restricting insurers' access to genetic test results, and how adverse selection might then affect the insurance market.

In a series of three papers, Macdonald et al. (2003a, b) and Gui et al. (2006) proposed models of BC and ovarian cancer (OC, also associated with BRCA1/2 mutations) in order to study critical illness (CI) and life insurance underwriting. Our purpose is to extend these models to IPI business in order to estimate premium ratings depending on genotype

or family history, and to model the potential cost of adverse selection. This will require a much more detailed model of BC, because: 
(a) IPI business allows for recoveries from sickness;

(b) BC might be diagnosed at one of several stages, each case leading to different treatments and recovery periods;

(c) BC can recur in several forms after initial treatment and each recurrence means another potential IPI claim; and

(d) the rate of recovery from $\mathrm{BC}$ varies with the choice of primary treatment which will depend on factors such as the stage of the cancer and the patient's genotype.

Therefore, the model must represent diagnosis, treatment, recovery and recurrence, and the relevant transition intensities must be estimated.

In this section, we summarise the relevant genetic epidemiology of $\mathrm{BC}$, and the model of IPI introduced by the Continuous Mortality Investigation Bureau (CMIB) in the UK. In Section 2, we propose an extension of this model to IPI business. In Sections 3-5, we introduce various stages of $\mathrm{BC}$ and the corresponding diagnostic approaches, based on which we propose a Markov model for the diagnosis of BC. Then for each type of diagnosis, we discuss treatment options, for the general population in Section 6 and for women with a BRCA1/2 mutation in Section 7. Based on the choice of treatment, we estimate relevant rates of recovery in Section 8. In Section 9, we estimate various rates of recurrence of BC. Finally, we summarize the life history model in Section 10.

\subsection{The Epidemiology of Breast Cancer}

$\mathrm{BC}$ is the most common cancer in women, accounting for $20 \%$ of all newly diagnosed cancers, and the lifetime risk of BC in the UK is 1 in 12 (Haites and Gregory, 2002). A history of $\mathrm{BC}$ among relatives has been found to indicate higher $\mathrm{BC}$ risk, and approximately 5-10\% of BC cases are thought to have inherited variants (Claus et al., 1991). Two BC susceptibility genes, BRCA1 and BRCA2, were identified in 1994 and 1995 respectively and are considered to be responsible for the majority of hereditary BC cases (Miki et al., 1994; Futreal et al., 1994; Wooster et al., 1995). BRCA1 and BRCA2 mutations are associated with a greatly increased lifetime risk of BC, with estimates of $59-87 \%$ for BRCA1 and 38-80\% for BRCA2 mutations (Ford et al., 1994; Easton et al., 1997; Struewing et al., 1997). See Gui et al. (2006) for a detailed description of the genetics of BC.

\subsection{Income Protection Insurance and Actuarial Models}

IPI replaces income lost during periods of disability. Usually, IPI policies have a specified deferred period (DP), and entitlement to benefit starts once a spell if illness exceeds the DP. Common DPs are 1 week, 4 weeks, 13 weeks or 26 weeks. Benefits cease when the policyholder recovers or dies, or when the policy expires. We will assume that policies expire at age 65 . Premiums are paid while benefits are not being received.

In CMIB Report (CMIR) No. 12 (1991), the CMIB proposed the multiple state model for IPI shown in Figure 1, governed by the transition intensities shown. These intensities depend on the policyholder's current age, $x$ and (in some cases) the duration of current sickness, $z$.

\section{An Extension of the IPI Model to Breast Cancer}

We need to distinguish BC from other illnesses and model the life histories accordingly. Our model must include the following, some of which depend on BRCA1/2 genotype: 


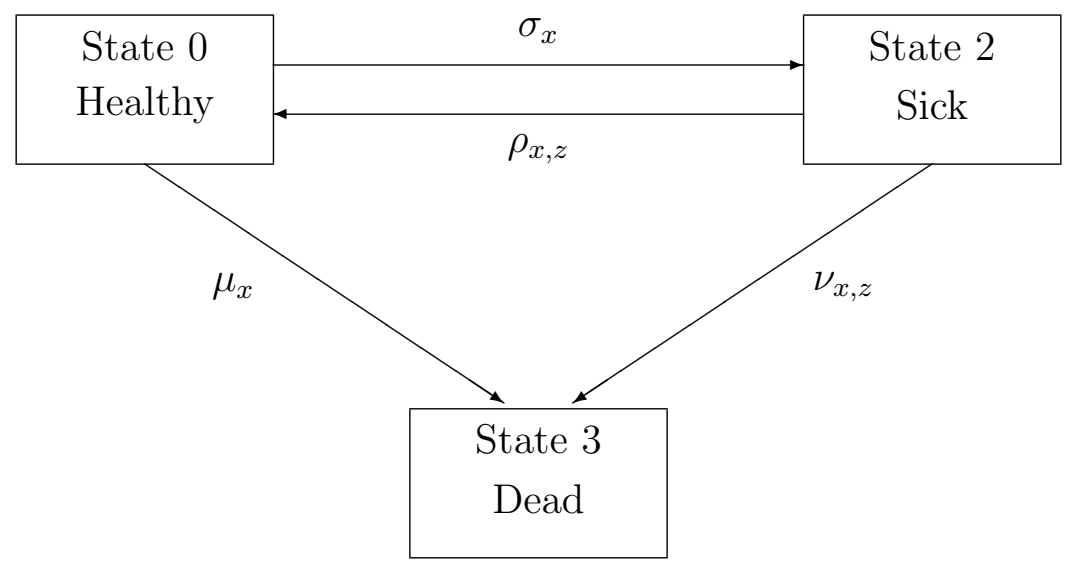

Figure 1: The CMIB's three-state semi-Markov model for sickness.

(a) onset of $\mathrm{BC}$ and progression between stages of $\mathrm{BC}$ (see Section 3);

(b) diagnosis, which can be at any stage of BC (see Sections 4 and 5);

(c) treatment depending on the stage and the patient's genotype (see Sections 6 and 7);

(d) recovery following the treatment (see Section 8);

(e) recurrence depending on initial treatment and the patient's genotype (see Section 9);

(f) further treatment and recovery; and

(g) death.

Figure 2 shows a continuous-time model of the life history of a woman with genotype $i$, in which the events above are represented as transitions between states. Possible genotypes $i$ are 'non-mutation carrier' or 'BRCA1/2 mutation carrier'. The 'other sickness' states represent all conditions leading to IPI claims except BC/OC. The model is semiMarkov, because some transition intensities depend on the duration in the current state, denoted $z$.

\section{Stages of Breast Cancer}

\subsection{Extent and Staging of Breast Cancer}

The 'stage' of BC at diagnosis describes the tumour spread and the level of deterioration. Hospitals and cancer registries use a variety of staging schemes designed to meet different needs. The prevalent schemes used today are:

(a) the American Joint Committee on Cancer's TNM system;

(b) the World Health Organization's numerical stages;

(c) the Surveillance, Epidemiology, and End Results (SEER)'s Extent of Disease; and

(d) the SEER's Summary Staging Guide. 


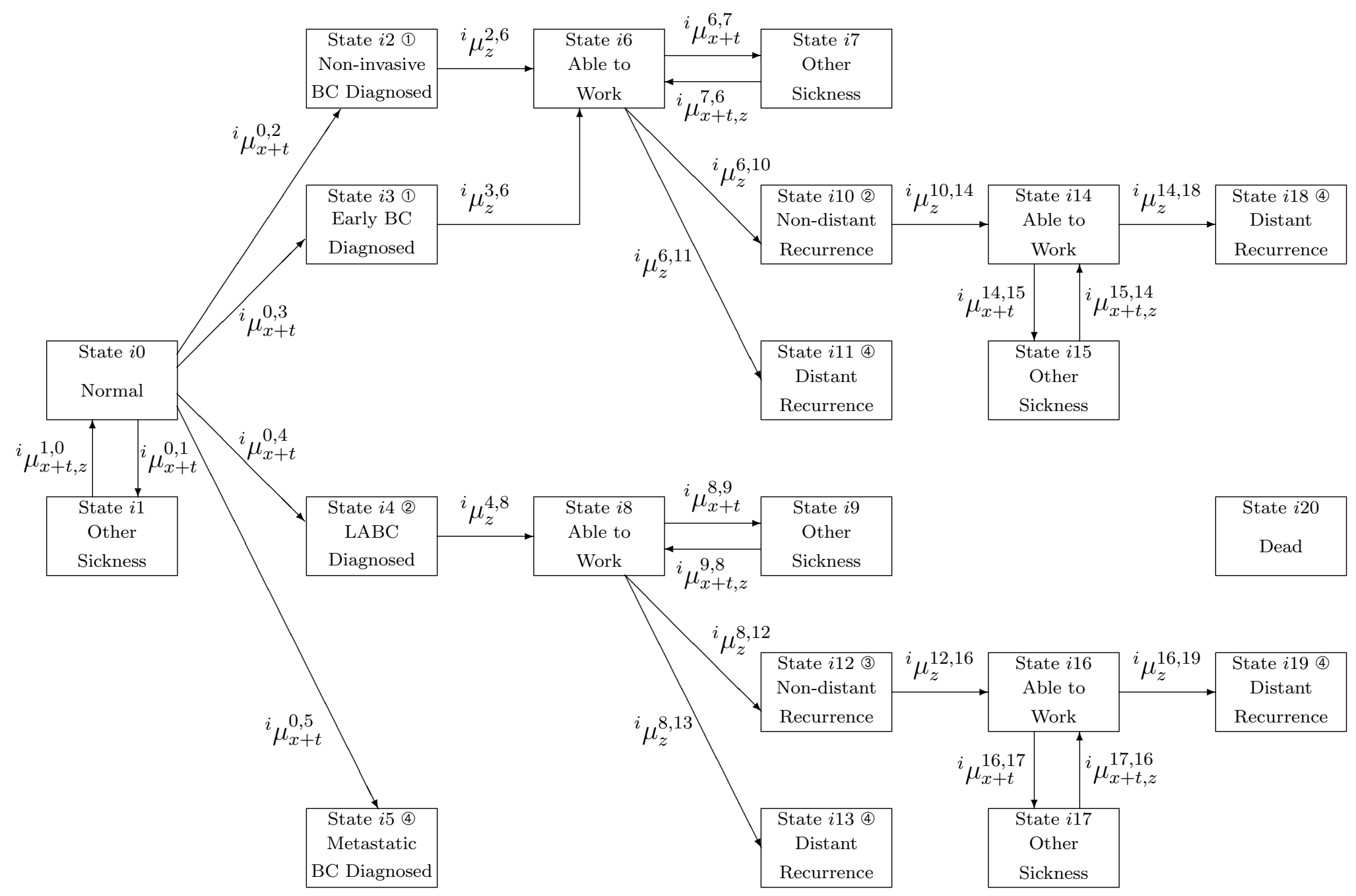

Figure 2: A semi-Markov model of the life history of a woman with genotype $i$ th. (1): treatment of BCS with radiotherapy; (2): combined treatment of mastectomy (bilateral mastectomy for BRCA1/2 mutation carriers), radiotherapy and chemotherapy; (3): chemotherapy for ipsilateral recurrence or combined treatment for contralateral recurrence; and (4): systematic treatment. Intensities are functions of current age, $x+t$, and duration in current state, $z$. 
See Seiffert (1993) and Young et al. (2001) for further descriptions of these systems and their mutual conversions. The SEER Program of the National Cancer Institute (NCI) is an authoritative source of information on cancer incidence and survival in the US. It currently collects population-based cancer registries covering about $26 \%$ of the US population and is considered to be the standard for quality around the world. Since the SEER's datasets are public, we use the staging system introduced by the SEER Summary Staging Guide, combined with current clinical categories (available at the websites of the NCI and the National Breast Cancer Centre) as follows. BC starts in the ducts or lobules of the breast, and according to its future development, it is categorized into two types: non-invasive and invasive.

\subsection{Non-invasive Breast Cancer}

Non-invasive BC means that cancer cells are confined to the ducts and do not invade the surrounding tissues. Ductal carcinoma in situ (DCIS) is the commonest form of noninvasive $\mathrm{BC}(90 \%)$. Lobular carcinoma in situ (LCIS) is less common and is considered to be a marker for increased BC risk. Because DCIS or LCIS are not usually palpable, most cases are found by routine screening with mammograms. Most women are not aware of any symptoms at the time of diagnosis. With early detection, the five-year survival rate for DCIS is nearly 100\%, provided that the cancer has not spread beyond the milk ducts.

\subsection{Invasive Breast Cancer}

Invasive $\mathrm{BC}$ means that cancer cells have spread from the ducts into the surrounding tissue. Invasive cancer cells sometimes spread outside the breast area through blood vessels, or through lymphatic vessels, that connect lymph nodes. So, cancer often spreads first to the axillary (armpit) lymph nodes and then to other areas of the body. It is usually categorized into three types, according to the invaded location.

(a) Early breast cancer: Cancer cells are confined in the breast and armpit area. Treatment is often successful; most women treated for early $\mathrm{BC}$ do not die from the disease.

(b) Locally advanced breast cancer ( $L A B C$ ): The tumour is larger than $4 \mathrm{~cm}$ and may have spread into the lymph nodes or other tissues next to the breast. It often indicates more aggressive cancer and it is much more likely than early BC to recur after primary treatments.

(c) Metastatic breast cancer (also known as advanced BC): Cancer cells have spread past the breast and axillary lymph nodes to other locations. $\mathrm{BC}$ has the potential to spread to almost any location. The most common is bone (75\%), followed by lung and liver. Present treatments for metastatic BC cannot cure the cancer; instead, they aim to relieve symptoms and extend life. Most women who die of $\mathrm{BC}$ have metastases, and these are directly responsible for the majority of $\mathrm{BC}$ deaths. The five-year survival rate is only 16\% (American Cancer Society, 1997).

\subsection{Recurrent Breast Cancer}

Occasionally BC can return after primary treatment (See Section 6), usually within two or three years, but sometimes many years later. Recurrent BCs fall into three categories as follows: 
(a) Ipsilateral recurrence: If a few cancerous cells are left in situ after surgery, local recurrence is possible. This is more failure of the primary treatment than true recurrence. A localized recurrence is much less serious than a regional recurrence, when a cancer has spread past the breast.

(b) Contralateral breast cancer: Contralateral BC means a tumour arises in the breast other than that first treated. It is generally considered to be a second primary cancer. Contralateral BC represents a much higher risk than ipsilateral recurrence since it has a new chance of metastasizing.

(c) Distant recurrence: A distant recurrence of BC is a metastatic BC. Its survival rate is considerably lower than for any other type of recurrence. Between $10 \%$ to $20 \%$ of recurrent cancers are ipsilateral; most are distant (Dunst et al., 2001).

\section{The Diagnosis of Breast Cancer}

\subsection{Diagnostic Approaches}

Procedures used to detect BC are mammography (X-rays), ultrasonography, magnetic resonance imaging (MRI), and positron emission tomography (PET). Mammography remains the most important for women with breast tissue that is not dense (Veronesi et al., 2005), and the others are mainly used to investigate abnormal mammograms. Mammography is used for screening and for diagnosis:

(a) Screening means examining asymptomatic women. Its goal is to detect cancer before it is palpable. In the UK, the NHS Breast Screening Programme was introduced in 1988 and invites all women aged 50-70 years for screening once every three years.

(b) Diagnostic mammography means examining a woman who either has a breast complaint or has an abnormality found during screening mammography.

In respect of the types of $\mathrm{BC}$ we described in Section 3, most non-invasive and some early cancers would not show any external symptoms and can only be detected by screening. The US Food and Drug Administration reported that mammography can find 85-90\% of BCs in women over 50 and could discover a lump up to two years before it can be felt. For the other types of $\mathrm{BC}$, the lump is palpable and obvious breast changes could be observed by clinical examination. Researchers have noted that some cancers, in particular $\mathrm{BC}$, are much more successfully treated when diagnosed early. Also, it has been shown that mortality from BC can be reduced by mass screening using mammography, because screening results in earlier diagnosis (Fagerberg et al., 1985; Tabar et al., 1985, 1987).

\subsection{The Participation Rate and Sensitivity}

The participation rate is the percentage of women in the population screened through a screening programme. Most published participation rates are population-based and reflect the level of medical development and completeness in that region. Participation rates vary by screening program, from $54 \%$ to $89 \%$, and Table 1 shows the recent participation rates in the UK.

The sensitivity of mammographic screening is the proportion of people with a tumour who have a positive test result for the breast cancer. It is a crucial measure of screening efficiency. BreastScreen Australia Monitoring Report 2000-2001 showed that the sensitivity 


\begin{tabular}{|c|c|c|c|c|}
\hline \multirow{2}{*}{$\begin{array}{l}\text { State } 0 \\
\text { Normal }\end{array}$} & $\lambda_{01}(x+t)$ & \multirow{2}{*}{$\begin{array}{l}\text { State } 1 \\
\text { PCDP }\end{array}$} & $\lambda_{12}(x+t)$ & \multirow{2}{*}{$\begin{array}{l}\text { State } 2 \\
\text { Clinical }\end{array}$} \\
\hline & & & & \\
\hline
\end{tabular}

Figure 3: Three-state Markov model of the progression of breast cancer.

of the Australian screening programme after first screen ranged from $86.1 \%$ in Tasmania to $96.6 \%$ in the Australian Capital Territory. On the other hand, the specificity of screening is the proportion of healthy persons correctly identified as not having a tumour. Specificity of less than $100 \%$ results in a certain proportion of mis-diagnoses, possibly leading to unnecessary treatments. We ignore its effect in what follows; therefore, to the extent that it might lead to additional claims under IPI contracts, we underestimate the cost attributable to BC.

\section{The Tumour Detection Model}

\subsection{The Tumour Progression Model}

The progression of a chronic disease after the introduction of a screening programme is often depicted by three states, represented by the Markov model in Figure 3:

(a) Every subject begins at age $x$ with no detectable disease, called 'normal'. Some subjects will develop the disease, while some will remain disease-free all their lives.

(b) A subject who develops the disease passes, at a random age $x+t_{1}$, to a state in which the disease is asymptomatic but can be detected by a screening test. This phase is called the 'preclinical screen-detectable phase' (PCDP). In terms of the categories we defined above, it means non-invasive breast cancer.

(c) Then, at a random age $x+t_{2}\left(t_{2} \geq t_{1}\right)$, the disease may become clinically symptomatic, in the absence of screening. This is defined as the time of diagnosis and the state entered is known as the 'clinical' state, or specifically, invasive breast cancer.

Several studies have used the Markov framework in Figure 3, and addressed the efficacy of mass mammographic screening programmes (Duffy et al., 1991, 1995, 1997; Tabar et al., 1996; Chen et al., 2002) among which, Duffy et al. $(1995,1997)$ and Chen et al. (2002) reported the progression rates explicitly. Duffy et al. $(1995,1997)$ assumed

Table 1: Published participation rates in the UK breast cancer screening program. Source: NHS Breast Screening Programme 2005 Review.

$\begin{array}{cccc}\text { Years } & \text { Rate (\%) } & \text { Years } & \text { Rate (\%) } \\ 1999-2000 & 75.4 & 2002-2003 & 74.7 \\ 2000-2001 & 75.0 & 2003-2004 & 75.2 \\ 2001-2002 & 75.5 & & \end{array}$


Table 2: Progression rates from Duffy et al. (1995, 1997).

\begin{tabular}{|c|c|c|c|}
\hline \multirow[t]{2}{*}{$\begin{array}{c}\text { Duffy et al. (1995) } \\
\text { All Ages }\end{array}$} & \multicolumn{3}{|c|}{ Duffy et al. (1997) } \\
\hline & $40-49$ & $50-59$ & $60-69$ \\
\hline 0.0052 & 0.00089 & 0.00155 & 0.0024 \\
\hline 0.43 & 0.26 & 0.18 & 0.20 \\
\hline
\end{tabular}

Table 3: MLEs of transition intensities of the model in Figure 3, from Chen et al. (2002).

$\begin{array}{lcc} & \text { MLE } & (95 \% \mathrm{CI}) \\ \hat{k} & 0.0000058 & (0.00000207-0.00000952) \\ \hat{\theta} & 2.4755 & (2.2496-2.7015) \\ \hat{\lambda}_{12} & 0.3153 & (0.04384-0.58676)\end{array}$

constant progression rates over time and age for simplicity. Their results are shown in Table 2 .

However, Chen et al. (2002) pointed out that constant progression rates might be unrealistic. The Armitage-Doll multi-stage model proposed that the age-specific incidence rates of many human adult carcinomas may be approximated by a Weibull form (Armitage and Doll (1961)). Therefore, for preclinical incidence, Chen et al. (2002) considered a Weibull distribution as follows:

$$
\lambda_{01}(x+t)=k \theta(x+t)^{\theta-1} .
$$

Since previous studies (Day et al., 1984) had shown the exponential distribution to be a good fit for the sojourn time in the PCDP state, Chen et al. (2002) kept $\lambda_{12}$ constant to simplify the estimation. Using data from the Taiwan Multicenter Cancer Screening Programme, MLEs were estimated as shown in Table 3.

\subsection{The Tumour Detection Model}

The model in Figure 3 is not adequate to model the life histories of applicants for IPI, for the following reasons:

(a) The model represents only the progression of BC, not detection or treatment. Screening will detect a proportion of tumours at PCDP. Furthermore, primary treatment, usually surgery, follows diagnosis. At this this time, the patient may be unable to work, triggering a IPI claim. Therefore, it is reasonable to modify the model to allow for tumour detection.

(b) The state 'clinical' referred to all invasive types of BC. However, BC is heterogeneous and treatment options (and outcomes) for each type vary greatly. For IPI, certain different types of BC should be treated separately.

In Figure 4, we show a continuous-time Markov model of the detection of BC. In fact, we can this model is just the diagnosis part of the model in Figure 2, where states have 


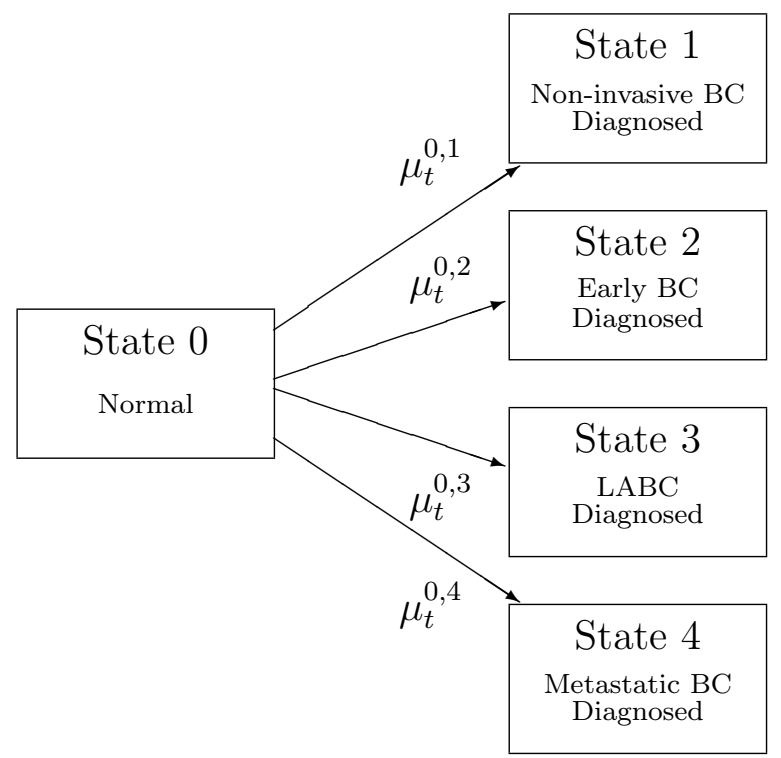

Figure 4: A Markov model of the detection of breast cancer.

the same names but different index numbers. To estimate the intensities, we use a variety of epidemiological studies of the transitions of interest, since no suitable estimates have been published, and we have no access to medical data.

(a) SEER rates of detection of invasive types: The SEER Program provides the SEER*Stat statistical software to utilize its public datasets. Based on SEER*Stat version 6.1.4, we selected the SEER 9 Regs Public-use database, with the registrations accepted between the yeasr 1973 and 2002. We only considered malignant BC and the first matching record for each person (hence removing recurrent $\mathrm{BC}$ ). Table 4 shows the rate of detection for each type of $\mathrm{BC}$, in which: (a) the 'Unstaged' column refers to records without this information or that are hard to classify; and (b) the 'rates' are based on the central exposure method. Pragmatically, we redistributed the 'Unstaged' column across the other three columns pro rata, see Table 5 .

However, these tables are based on US cancer registries, and we ought to compare US and UK experiences. In Figure 5, we compare the incidence rates of $\mathrm{BC}$ based on the SEER dataset, and on cancer registries in England and Wales between 1990-1992 (O.N.S., 1999), with fitted onset rates from Macdonald et al. (2003a). The UK fitted rates drop unusually afterwards, because of the introduction in 1988 of the NHS Breast Screening Programme for women above age 50 (described in Section 4.1) In the US, cancer registration started before the introduction of any public screening program, hence a considerable proportion of BCs were diagnosed by traditional clinical approaches. Screening results in tumours being diagnosed earlier, before they become palpable. Nevertheless, the rates tally closely before age 50 .

(b) The rate of detection of non-invasive breast cancer: Unfortunately, SEER data do not report onset of non-invasive $\mathrm{BC}$, so we represent the detection rate by modifying the PCDP rate $\lambda_{01}(t)$ in the tumour progression model in Figure 3. As we described in 


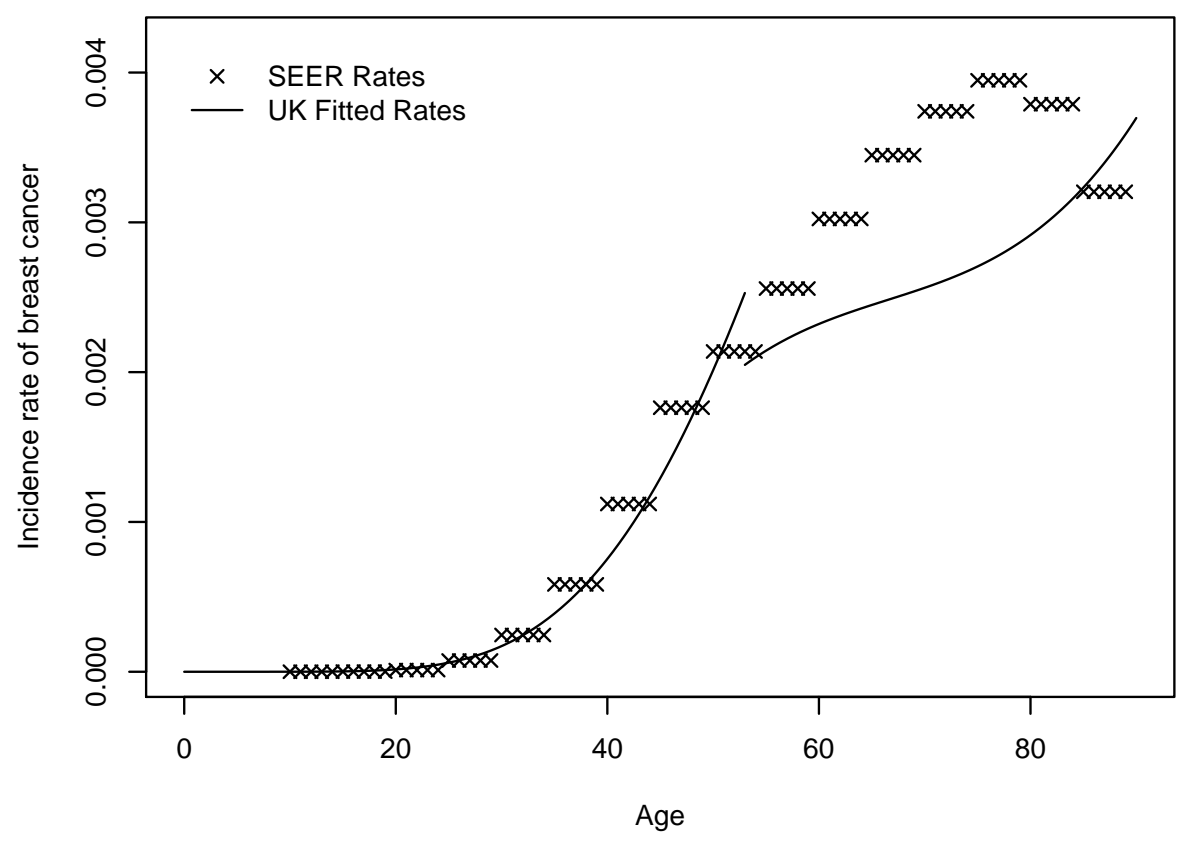

Figure 5: Comparison of incidence rates of breast cancer, based on the SEER 9 Regs Public-use (1973-2002) database and UK fitted rates from Macdonald et al. (2003a).

Table 4: The detection rates of invasive types of breast cancer, based on SEER 9 Regs Public-use database. COUNT is the number of BC cases detected during the age interval and TE is the total exposure to risk. Rates are per 100,000 women years.

\begin{tabular}{cccccccccc} 
& \multicolumn{2}{c}{ Early BC } & \multicolumn{2}{c}{ LABC } & \multicolumn{3}{c}{ Metastatic BC } & Unstaged & \\
Age & COUNT & Rate & COUNT & Rate & COUNT & Rate & COUNT & Rate & TE \\
$20-24$ & 150 & 0.6 & 136 & 0.5 & 18 & 0.1 & 21 & 0.1 & $27,236,827$ \\
$25-29$ & 1,068 & 3.7 & 990 & 3.4 & 107 & 0.4 & 107 & 0.4 & $28,927,095$ \\
$30-34$ & 3,554 & 12.3 & 3,119 & 10.8 & 412 & 1.4 & 283 & 1.0 & $28,864,320$ \\
$35-39$ & 8,088 & 30.4 & 6,676 & 25.1 & 762 & 2.9 & 522 & 2.0 & $26,623,302$ \\
$40-44$ & 14,927 & 62.2 & 10,669 & 44.4 & 1,297 & 5.4 & 746 & 3.1 & $24,014,180$ \\
$45-49$ & 20,820 & 99.0 & 14,335 & 68.2 & 1,904 & 9.1 & 1,026 & 4.9 & $21,025,117$ \\
$50-54$ & 22,876 & 121.7 & 14,990 & 79.7 & 2,335 & 12.4 & 1,047 & 5.6 & $18,802,463$ \\
$55-59$ & 24,074 & 146.3 & 15,236 & 92.6 & 2,783 & 16.9 & 1,183 & 7.2 & $16,455,339$ \\
$60-64$ & 26,061 & 178.4 & 15,017 & 102.8 & 3,074 & 21.0 & 1,266 & 8.7 & $14,606,912$ \\
$65-69$ & 28,288 & 214.3 & 14,084 & 106.7 & 3,150 & 23.9 & 1,338 & 10.1 & $13,200,388$ \\
$70-74$ & 26,958 & 238.4 & 12,525 & 110.8 & 2,815 & 24.9 & 1,535 & 13.6 & $11,306,450$ \\
$75-79$ & 23,333 & 255.6 & 10,287 & 112.7 & $2,, 426$ & 26.6 & 1,597 & 17.5 & $9,129,582$ \\
$80-84$ & 15,605 & 244.1 & 6,875 & 107.5 & 1,745 & 27.3 & 1,789 & 28.0 & $6,394,173$ \\
$85+$ & 11,381 & 197.7 & 5,506 & 95.7 & 1,557 & 27.0 & 3,360 & 58.4 & $5,756,315$
\end{tabular}


Table 5: The modified detection rates of invasive types of breast cancer, based on SEER 9 Regs Public-use database. COUNT is the number of breast cancer detected during the age interval and TE is the total exposure to risk. Rates are per 100,000 women years.

\begin{tabular}{cccccccc} 
& \multicolumn{2}{c}{ Early BC } & \multicolumn{2}{c}{ LABC } & \multicolumn{3}{c}{ Metastatic BC } \\
Age & COUNT & Rate & COUNT & Rate & COUNT & Rate & TE \\
$20-24$ & 160 & 0.6 & 145 & 0.5 & 19 & 0.1 & $27,236,827$ \\
$25-29$ & 1,121 & 3.9 & 1,039 & 3.6 & 112 & 0.4 & $28,927,095$ \\
$30-34$ & 3,696 & 12.8 & 3,244 & 11.2 & 428 & 1.5 & $28,864,320$ \\
$35-39$ & 8,360 & 31.4 & 6,900 & 25.9 & 788 & 3.0 & $26,623,302$ \\
$40-44$ & 15,341 & 63.9 & 10,965 & 45.7 & 1,333 & 5.6 & $24,014,180$ \\
$45-49$ & 21,396 & 101.8 & 14,732 & 70.1 & 1,957 & 9.3 & $21,025,117$ \\
$50-54$ & 23,472 & 124.8 & 15,380 & 81.8 & 2,396 & 12.7 & $18,802,463$ \\
$55-59$ & 24,751 & 150.4 & 15,664 & 95.2 & 2,861 & 17.4 & $16,455,339$ \\
$60-64$ & 26,808 & 183.5 & 15,448 & 105.8 & 3,162 & 21.6 & $14,606,912$ \\
$65-69$ & 29,119 & 220.6 & 14,498 & 109.8 & 3,243 & 24.6 & $13,200,388$ \\
$70-74$ & 27,936 & 247.1 & 12,980 & 114.8 & 2,917 & 25.8 & $11,306,450$ \\
$75-79$ & 24,367 & 266.9 & 10,743 & 117.7 & 2,533 & 27.8 & $9,129,582$ \\
$80-84$ & 16,757 & 262.1 & 7,383 & 115.5 & 1,874 & 29.3 & $6,394,173$ \\
$85+$ & 13,454 & 233.7 & 6,509 & 113.1 & 1,841 & 32.0 & $5,756,315$
\end{tabular}

Section 4 , women with a tumour who attend an organized screening programme would or would not be detected as carrying a tumour depending on the sensitivity of the test. Therefore, the detection rate of non-invasive BC $\left(\mu_{x+t}^{0,2}\right.$ in Figure 2$)$ is estimated by multiplying $\lambda_{01}(t)$ by the participation rate and the sensitivity. $\lambda_{01}(t)$ is estimated from Chen et al. (2002) using the Weibull form in Equation (1), the participation rate is estimated as 0.75 and the sensitivity as 0.9 , as described in Section 4.2.

For BRCA1/2 mutation carriers, the detection rates of specific types of $\mathrm{BC}$ were not reported in the medical literature. However, the overall onset rates of BC (all types) for mutation carriers were reported by Antoniou et al. (2003) and fitted to truncated Gamma functions by Gui et al. (2006) as follows:

$$
\begin{aligned}
\mu_{x}^{B C, B R C A 1} & =\frac{1.39}{\Gamma(12.2)}\left(0.21^{12.2} e^{-0.21 x} x^{11.2}\right) \\
\mu_{x}^{B C, B R C A 2} & =\frac{1.58}{\Gamma(8.37)}\left(0.10^{8.37} e^{-0.1 x} x^{7.37}\right)
\end{aligned}
$$

We assume these onset rates decompose into of each type of $\mathrm{BC}$ as in the general population. For example, for BRCA1 mutation carriers, the detection rate of non-invasive $\mathrm{BC}$ is:

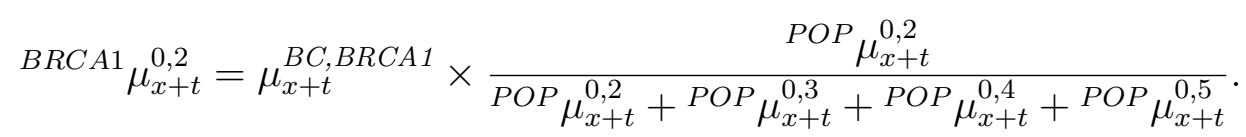


Table 6: Twenty-year results of an Italian randomized trial of breast cancer surgery. Source: Veronesi et al. (2002).

$\begin{array}{lcc} & \text { Radical mastectomy } & \text { Breast-conserving surgery } \\ \text { Number } & 349 & 352 \\ \text { Ipsilateral recurrence } & 8(2.3 \%) & 30(8.6 \%) \\ \text { Contralateral recurrence } & 34(9.7 \%) & 29(8.3 \%) \\ \text { Distant recurrence } & 83(24 \%) & 82(24 \%) \\ \text { Other primary cancers } & 30(8.6 \%) & 31(8.9 \%) \\ \text { Death from BC } & 86(25 \%) & 91(26 \%) \\ \text { All deaths } & 152(44 \%) & 156(44 \%)\end{array}$

\section{Breast Cancer Treatment I: The General Population}

Most women who have been diagnosed with $\mathrm{BC}$ will be treated immediately or after a short time. The choice of treatment will depend on a number of factors, including the size and location of the tumour, the stage of the cancer, and histological results.

\subsection{Forms of Treatment}

There are three common forms of treatment: surgery, radiotherapy and chemotherapy. Most women with breast cancer will undergo surgery as a primary treatment. The standard types of breast surgery include: (a) breast conservation surgery (BCS; also known as lumpectomy) which removes only the cancerous lump (and possibly some surrounding tissue); and (b) mastectomy which removes the whole breast. In addition to surgery, chemotherapy and radiotherapy are often offered as adjuvant treatment. See Veronesi et al. (2005) and the Steering Committee on Clinical Practice Guidelines for the Care and Treatment of Breast Cancer $(1998,2001)$ for further details.

\subsection{Mastectomy versus $B C S$}

The radical mastectomy introduced by Halsted (Halsted et al., 1898) was the treatment of choice for 80 years. Then a randomized study found that mastectomy and BCS had equal survival rates (Veronesi et al., 1977, 1981). Finally, Veronesi et al. (2002) and Fisher et al. (2002) reported on 20 years of follow-up and confirmed that BCS with radiotherapy produced similar results to radical mastectomy, as shown in Tables 6 and 7 . Now, BCS with subsequent radiation has become the favoured treatment for women with non-invasive or early BC.

\subsection{Treatment Options}

Here we outline prevalent treatments, ignoring exceptions and special cases.

(a) Non-invasive and early breast cancer: The aim is to remove the cancer from the breast and armpit area by surgery. According to the Canadian Medical Association (Steering Committee on Clinical Practice Guidelines for the Care and Treatment of Breast Cancer, 1998, 2001), BCS with radiotherapy is generally recommended unless there are special reasons for selecting mastectomy. 
Table 7: Twenty-year results of a US randomized trial of breast cancer surgery. Source: Fisher et al. (2002).

$\begin{array}{rcc}\text { Number } & \text { Radical mastectomy } & \text { Breast-conserving surgery } \\ \text { Ipsilateral recurrence } & 87(14.8 \%) & 628 \\ \leq 10 \text { year } & 82(13.9 \%) & 51(8.1 \%) \\ >10 \text { year } & 7(0.9 \%) & 42(6.7 \%) \\ \text { Contralateral recurrence } & 50(8.5) & 9(1.4 \%) \\ \leq 10 \text { year } & 30(5.1 \%) & 59(9.4 \%) \\ >10 \text { year } & 20(3.4 \%) & 40(6.4 \%) \\ \leq 10 \text { year } & 117(19.9 \%) & 19(3 \%) \\ >10 \text { year } & 15(2.5 \%) & 163(26 \%) \\ \text { Distant recurrence } & 43(7.3 \%) & 23(3.7 \%) \\ \text { Other primary cancers } & 312(53 \%) & 49(7.8 \%) \\ \text { Death from BC } & 371(63 \%) & 322(51 \%) \\ \text { All deaths } & & 391(62 \%)\end{array}$

(b) Locally advanced breast cancer: The local excision of the tumour is not a wise choice because cancer cells may exist throughout the breast and surrounding area. Standard therapy is mastectomy usually with radiotherapy and chemotherapy. The National Institutes of Health has recommended that most women with LABC be offered chemotherapy (NIH Consensus Development Panel, 2000). Therefore, we consider mastectomy with adjuvant radiotherapy and chemotherapy to be the general treatment for patients at this stage.

(c) Metastatic breast cancer: Present medical science generally cannot cure metastatic cancer. The speed of diffusion and reproductive ability of metastatic cancer cells limit the value of surgery. Thus, the purpose of treatment turns to pain relief and survival time. According to the Steering Committee on Clinical Practice Guidelines for the Care and Treatment of Breast Cancer (1998, 2001), women diagnosed with metastatic BC will usually be recommended to have systematic treatment which may involve one or more general anti-cancer treatments, and one or more specific treatments depending on the cancer's location and symptoms. General anti-cancer treatments are treatments that work on the whole body to control the size of spread of the cancer. They include chemotherapy and hormotherapy.

(d) Non-distant recurrence: Both ipsilateral and contralateral recurrences imply a second chance for metastasis. Treatment often depends on how the initial surgery was performed:

(1) In most trials, a recurrent BC after BCS is treated by mastectomy despite a policy of re-excision followed by radiation. For example, the Swedish Trial (UppsalaOrebro Breast Cancer Study Group, 1990) reported an overall mastectomy rate of $70 \%$ for ipsilateral recurrence after initial BCS. So in this study, we assume mastectomy with chemotherapy would be performed for recurrent BC treated initially by BCS. 
(2) If ipsilateral recurrence appears in patients who have already undergone mastectomy, it means cancer cells have invaded outside the breast area, hence there is much higher risk. In this case, we assume chemotherapy would be offered to reduce the risks of metastasis. Otherwise, if the recurrence is diagnosed in the contralateral breast, which represents a second primary tumour, a combination of mastectomy, radiotherapy and chemotherapy would be performed on the affected breast (Steering Committee on Clinical Practice Guidelines for the Care and Treatment of Breast Cancer, 1998, 2001).

(e) Distant recurrence: The treatment would be the same as for metastatic BC.

\section{Breast Cancer Treatment II: BRCA1/2 Mutation Carriers}

\subsection{Increased Risk Associated with BRCA1 and BRCA2 Mutations}

BRCA1 and BRCA2 mutations are considered to account for approximately $5-10 \%$ of breast cancers detected, with most occurring in women younger than age 50 years (Claus et al., 1996). BRCA1 or BRCA2 mutation carriers have a cumulative risk of invasive $\mathrm{BC}$ of $64 \%$ by age 70 (Scott et al., 2003), and those with BC have a cumulative risk of contralateral BC of $50-60 \%$ by age 70 (The Breast Cancer Linkage Consortium, 1999) versus $2 \%$ for the general population (Nicolletto et al., 2001). Verhoog et al. $(1998,1999)$ reported that in a five-year follow-up, the rates of contralateral BC in BRCA1 or BRCA2 mutation carriers were $19 \%$ and $12 \%$ respectively, compared with rates of $5 \%$ and $2 \%$ respectively in age-matched non-carriers. Mutation carriers also have an increased risk of ipsilateral recurrence (Turner et al., 1999; Haffy et al., 2002).

\subsection{Treatment for Unaffected Carriers: Bilateral Prophylactic Mastectomy}

Given the high risks described above, unaffected mutation carriers may consider preventative management to reduce their risk of $\mathrm{BC}$. Three strategies are available: surveillance, chemoprevention, and prophylactic surgeries. The decision is difficult, and may differ based on cultural attitudes and how the options are presented. It generally involves counselling and may include psychological assessment. More and more BRCA1/2 carriers are now seriously considering and/or undergoing bilateral prophylactic mastectomy (BPM). In the Dutch study by Meijers-Heijboer et al. (2000), 51\% (35/68) of unaffected BRCA1/2 mutation carriers chose BPM. A similar rate of uptake of $48 \%$ was reported by Lalloo et al. (2000). However, in the UK, the uptake rate seems not so high, Evans et al. (1999) showed that $11 \%$ of women at $40 \%$ lifetime risk of BC proceeded with BPM. In this study, we assume a medium uptake rate of $20 \%$.

Retrospective and prospective studies of BPM have reported a high degree of risk reduction:

(1) Rebbeck et al. (2004): The patients were 483 women with BRCA1/2 mutations, of whom 105 underwent BPM. In an average of 6 years of follow-up, BC was diagnosed in $2(1.9 \%)$ of these 105 women and $184(48.7 \%)$ of the 378 controls. The risk reduction was up to about $95 \%$.

(2) Hartmann et al. (2001): In a selected high-risk group of 214 women, 26 were identified as BRCA1/2 mutation carriers and underwent BPM. After a median of 13.4 years follow up, no BC was diagnosed. The risk reduction was $100 \%$. 
(3) Meijers-Heijboer et al. (2001): No BC was detected in the 76 BRCA1/2 mutation carriers who underwent BPM, compared with 8 diagnosed BCs in 63 controls.

In the study of Rebbeck et al. (2004) described above, the 105 women who underwent BPM were at risk for 557.36 person-years, giving a rate of onset of BC of 0.0036 per annum, similar to that for the general population shown in Figure 5. Furthermore, there seems to be no significant difference in survival rates after onset between BRCA1/2 mutation carriers and non-carriers (Verhoog et al., 1998, 1999; El-Tamer et al., 2004). From Hartmann et al. (2001), we deduce an average annual mortality rate from BC of 0.00067 (2 events/2970 person-years) for mutation carriers who underwent BPM, which is also similar to the level in the general population. Therefore, in this study, we assume that unaffected BRCA1/2 mutation carriers who undergo BPM are considered as being exposed to the population risk of $\mathrm{BC}$.

\subsection{Treatment for Affected Carriers: BCS versus Bilateral Mastectomy}

The optimal management for BCs related to BRCA1/2 mutations remains controversial. General procedures are listed as follows, but treatment varies according to region, cultural background and other practical factors.

(a) Non-invasive and early breast cancer: BCS with radiotherapy has been definitely established as an acceptable standard of management for the majority of women with non-invasive and early BC (Veronesi et al., 2002; Fisher et al., 2002). However, its efficacy for women with hereditary BC is still debated. Some reports show that 5-year risks of ipsilateral recurrence are similar to those experienced by non-carriers (Foulkes et al., 1997; Pierce et al., 2003; Robson et al., 2004), while others report an increased risk (Turner et al., 1999; Haffy et al., 2002). This suspected excess risk, as well as the confirmed high risk of contralateral recurrence, has led many patients strongly to consider bilateral mastectomy (BM) at the time of diagnosis. This is a hard decision especially for younger women. Unfortunately most hereditary $\mathrm{BC}$ occurs in this age group. Therefore, BCS with radiotherapy is still a prevalent option. Schwartz et al. (2004) reported 194 patients who underwent genetic testing at the time of $\mathrm{BC}$ diagnosis, of whom 31 tested positive. Of these, 15 (48.4\%) chose BM, another $15(48.4 \%)$ chose BCS with radiotherapy, and only one woman chose ipsilateral mastectomy. BCS with radiotherapy was chosen by the majority of noncarriers.

(b) Locally advanced breast cancer: At this stage, treatment concentrates on reducing the risk of metastasis and improving survival. Most patients and clinicians would chose BM. Reconstructive surgery would be performed immediately and followed by 4-6 months of chemotherapy.

(c) Metastatic breast cancer: Once the cancer has spread to other areas, no difference of treatment seems to exist between non-carriers and carriers.

\section{Treatment Procedures and Recovery}

During the session of treatment, patients are unable to work and may claim IPI benefit (after the DP). Therefore, we need to estimate the distribution of the duration of disability based on various treatment options. 


\subsection{Treatment Procedures}

We have the following information in respect of the treatments described in Sections 6 and 7 :

(a) BCS with radiotherapy: Veronesi et al. (2005) reported that BCS was typically followed 3-4 weeks later by at least 5-6 weeks of radiotherapy + . In addition, we assume 1-2 weeks of preparation before surgery and 1-2 weeks of rest after radiotherapy. Therefore, we estimate a total of 10-14 weeks of disability.

(b) Mastectomy with immediate breast reconstruction surgery: The majority of women who choose mastectomy opt for reconstructive surgery immediately afterward. Robson et al. (2004) suggested an average 6-8 weeks of disability.

(c) Adjuvant chemotherapy: Chemotherapy is usually given in 'cycles', consisting of a short period of treatment, followed by a rest period. The length of treatment and rest periods will depend on the type of $\mathrm{BC}$ and the type of chemotherapy. Normally, the duration would be 4-6 months starting 4-6 weeks after surgery. The most prevalent chemotherapy treatments for women with early BC are cyclophosphamide, methotrexate and fluorouracil (CMF) combination chemotherapy offered for the duration of 6 months (Colleoni et al., 2002). The Early Breast Cancer Trialists' Collaborative Group (EBCTCG) compared the standard protocol (4-6 months) with double the duration (8-12 months) and found no additional benefit from the latter (EBCTCG, 1998). Therefore, we assume an overall disability period of 4-6 months for women who receive chemotherapy.

(d) Systematic treatment: In general, once a metastatic BC is diagnosed, the patient will receive systematic treatment until death. Frequent treatments with long periods and serious physical condition normally prevents them returning to work. Therefore, we assume no recovery for a woman with metastatic BC.

\subsection{Rates of Recovery based on Treatment Options}

In the IPI model, 'recovery' from BC means claim termination through being able to work again. In the model in Figure 2, these recoveries are represented by transition intensities from BC states into 'Able to Work' states. We assume these intensities are functions of the sickness duration $z$ only, because treatment procedures are highly standardised below age 70, by which time most IPI policies have expired. Therefore, we use recovery rates from $\mathrm{BC}$ as follows, based on treatment options:

(1) For women who undergo BCS with radiotherapy, the average period of disability is 10-14 weeks. Here, we assume that patients return to work evenly between the 10th week and the 14th week after treatment, which implies a uniform distribution of the sickness duration $z$ between 10 weeks and 14 weeks. Then the recovery rates after BCS with radiotherapy, ${ }^{i} \mu_{z}^{2,6}$ and ${ }^{i} \mu_{z}^{3,6}$, could be expressed as:

$$
\mu_{z}^{\text {Recovery, } B C S}= \begin{cases}\frac{26}{7-26 z} & 10 / w \leq z<14 / w \\ 0 & \text { otherwise }\end{cases}
$$

where $z$ is duration in years, and we assume there are $w=52$ weeks in a year. 
(2) For women who undergo the combined treatment of mastectomy, radiotherapy and adjuvant chemotherapy, the disability period includes the average $6-8$ weeks taken up by mastectomy with immediate breast reconstruction surgery, plus the general duration of chemotherapy of 4-6 months starting 4-6 weeks after surgery and then followed by $5-6$ weeks of radiotherapy starting 2-4 weeks after chemotherapy ends. Similarly to the above, the recovery rates, ${ }^{i} \mu_{z}^{4,8},{ }^{i} \mu_{z}^{10,14}$ and ${ }^{i} \mu_{z}^{12,16}$, are:

$$
\mu_{z}^{\text {Recovery, } M A S}= \begin{cases}\frac{13}{12-13 z} & 33 / w \leq z<48 / w \\ 0 & \text { otherwise }\end{cases}
$$

\section{Rates of Breast Cancer Recurrence}

\subsection{Recurrence of Breast Cancer}

$\mathrm{BC}$ may recur after initial treatment. In the model in Figure 2, these recurrences are represented by transitions from 'Able to Work' states into recurrence states. We assume the corresponding intensities depend only on the time since the end of last treatment, $z$, which is equivalent to the duration in the 'Able to Work' state, because we assume that women will immediately return to work after the end of treatment.

Recurrence rates vary between non-carriers and BRCA1/2 mutation carriers, according to the type of recurrence and primary treatment. Let $\mu_{z}^{R, T, G}$ represent the rate of recurrence of type $R$, for a woman of genotype $G, z$ years after primary treatment of type $T$. For example, $\mu_{z}^{I R, B C S, N C}$ is the rate of onset of ipsilateral recurrence $z$ years since primary treatment of BCS for a non-mutation carrier. Chappuis et al. (2002) gave a comprehensive review of recent studies of $\mathrm{BC}$ recurrence for both the general population and BRCA1/2 mutation carriers. We have investigated most of these and we choose Robson et al. (2004) as our basic data source for two reasons:

(a) All patients underwent genetic tests for a BRCA1/2 mutation, and hence their genotypes were clear.

(b) Follow up was from diagnosis with a median of 10 years, which provides reliable evidence for long-term effects.

\subsection{Recurrence Rates after Primary Treatment of BCS for Early Breast Cancer}

The data used in Robson et al. (2004) include two similarly designed historical cohorts from the Memorial Sloan-Kettering Cancer Center in New York and the Sir Mortimer B. Davis-Jewish General Hospital in Montreal. There were 496 women in the database, of whom 42 were found to carry a BRCA1 mutation, 13 were found to carry a BRCA2 mutation and 1 was found to carry both BRCA1 and BRCA2 mutations. The latter woman was excluded leaving 495 subjects. Twenty-four women underwent contralateral BCS during the period of the study. Thus, for the purposes of determining the rate of ipsilateral cancer, there were 519 breasts at risk. All 495 patients underwent BCS plus radiotherapy between 1980 and 1995 and a summary of BC recurrence is shown in Table 8, after a median of 10 years of follow-up. 
Table 8: Ten-year follow-up of breast cancer recurrence after primary treatment of BCS. Source: Robson et al. (2004)

$\begin{array}{lccc} & \text { Non-Carriers } & \text { BRCA1 Carriers } & \text { BRCA2 Carriers } \\ \text { Number of patients } & 440 & 42 & 13 \\ \text { Ipsilateral recurrence } & 40(9.1 \%) & 4(9.5 \%) & 0(0.0 \%) \\ \text { Contralateral recurrence } & 37(8.4 \%) & 15(28.6 \%) & 3(23.1 \%) \\ \text { Distant recurrence } & 82(18.6 \%) & 19(45.2 \%) & 3(23.1 \%)\end{array}$

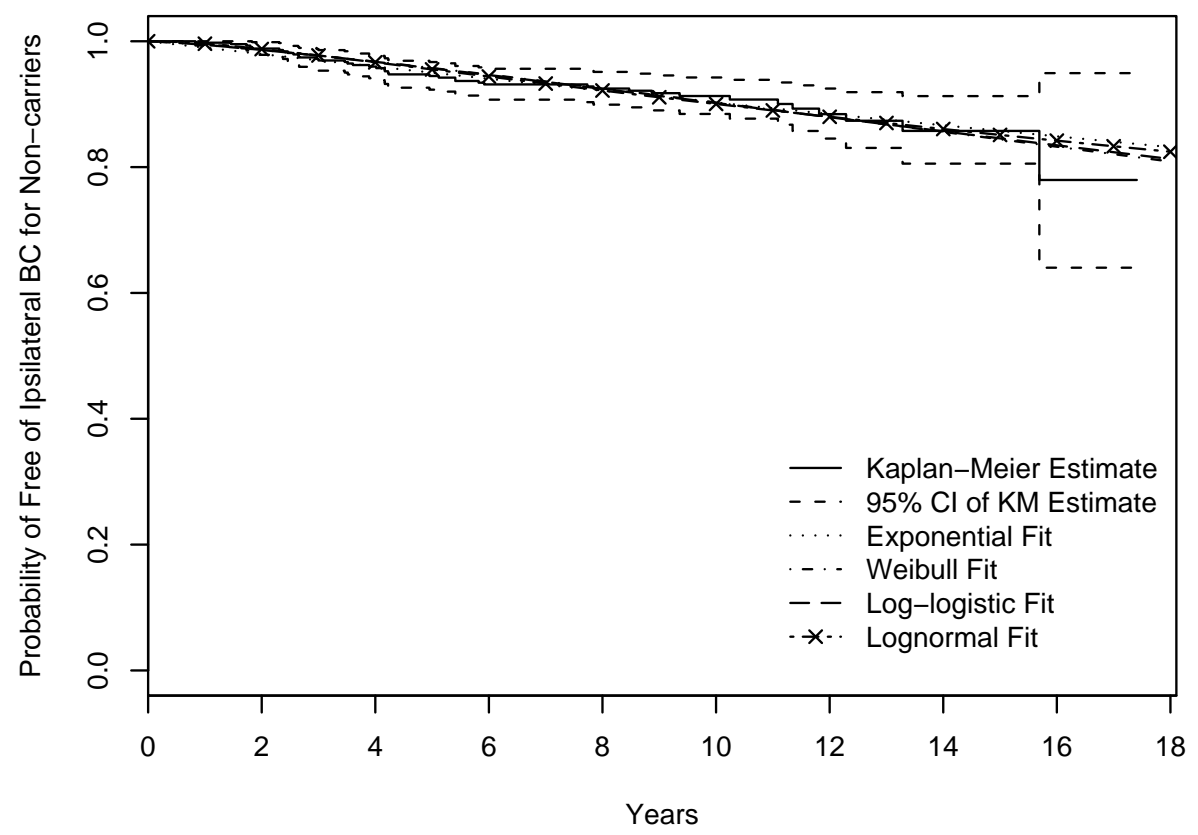

Figure 6: Non-parametric and parametric estimates for the probability of survival free of ipsilateral recurrence for non-carriers of BRCA1/2 mutations.

Let $T$ denote the time to the event of interest, namely recurrence. We wish to estimate the distribution of $T$. We consider the Kaplan-Meier (K-M) non-parametric approach, and four parametric models: exponential, Weibull, log-logistic and log-normal. These four models share the property that $\log T$ is a member of the location and scale family of distributions with the following features: (a) the distributions of $T$ have two parameters: scale $\lambda$ and shape $\alpha$, while the distributions of $\log T$ have two parameters: location $\mu$ and scale $\sigma$, where: $\mu=-\log \lambda$ and $\sigma=1 / \alpha$; and (b) all can be expressed in $\log$-linear form: $\log T=\mu+\sigma Z$, where $Z$ is that member of the family containing $\log T$ with $\mu=0$ and $\sigma=1$.

For non-carriers and BRCA1/2 mutation carriers, we fit models to ipsilateral, contralateral and distant recurrences, using non-parametric K-M estimates and the four parametric models described above.

(a) For non-carriers, Figure 6 shows the Kaplan-Meier and parametric estimates of the 


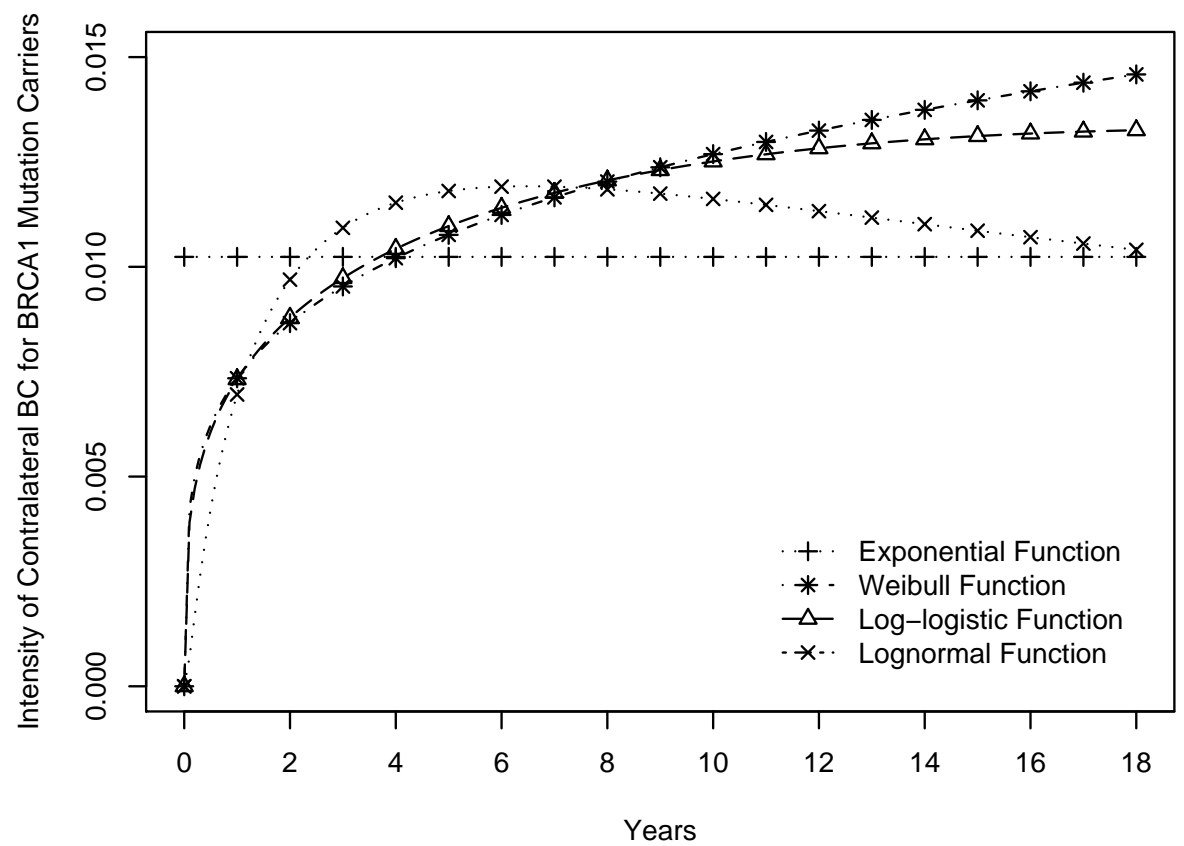

Figure 7: Parametric rates of onset of ipsilateral recurrence for non-carriers of BRCA1/2 mutations.
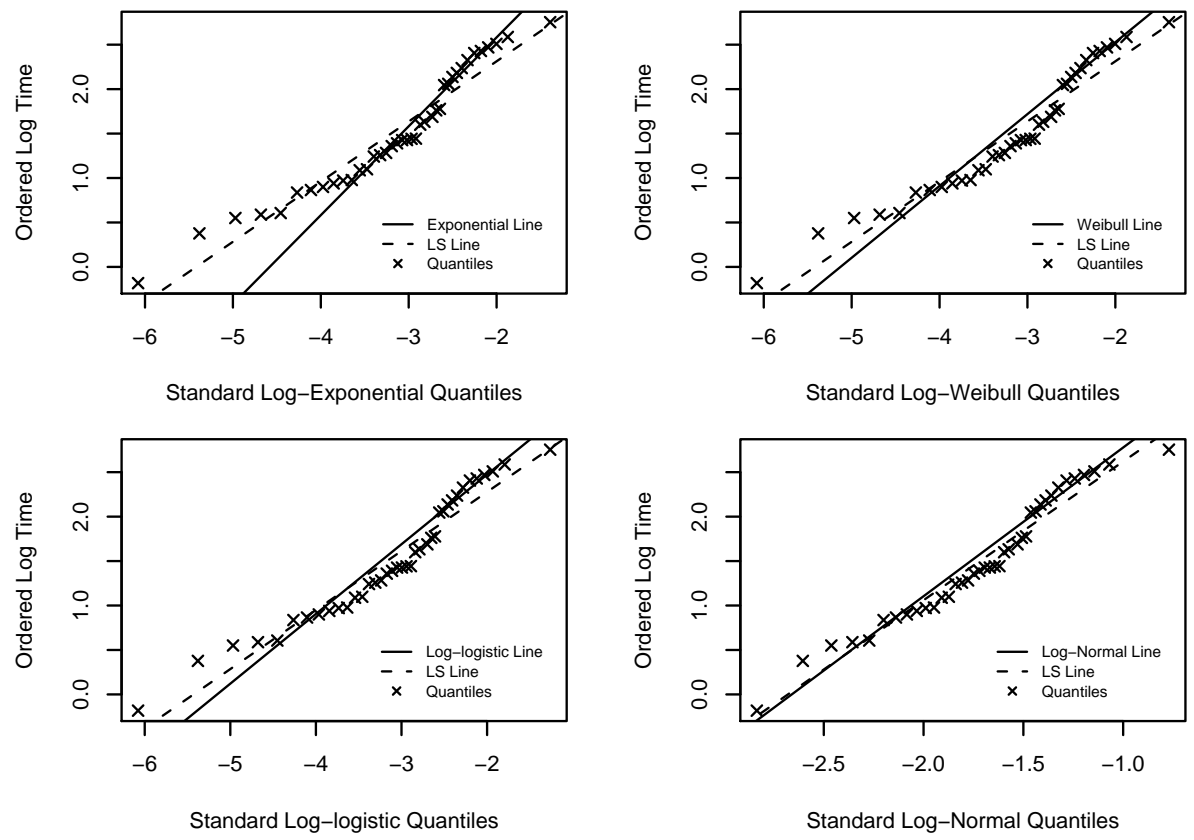

Figure 8: Q-Q plots to test model adequacy of ipsilateral recurrence for non-carriers of BRCA1/2 mutations. 

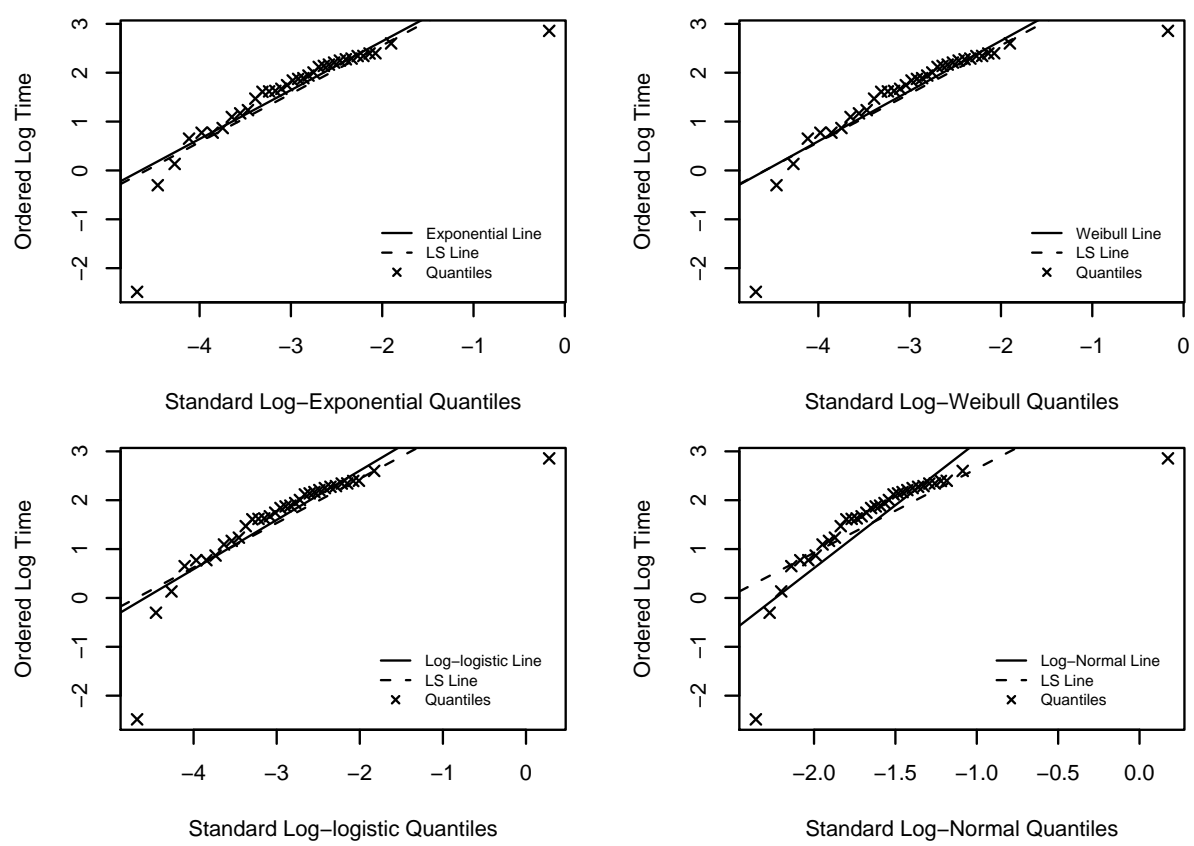

Figure 9: Q-Q plots to test model adequacy of contralateral recurrence for non-carriers of BRCA1/2 mutations.

probability of survival free of ipsilateral recurrence. Figure 7 shows the corresponding rates of onset of ipsilateral recurrence. Figure 8 shows the quartile-quartile (Q-Q) plots for each parametric distribution to test the model fit, where the solid lines are constructed with the estimates $\hat{\mu}$ and $\hat{\sigma}$; and the dashed lines are least squares lines of the quartile points. On consideration of these, we choose the log-normal distribution, with $\hat{\mu}=4.4501$ and $\hat{\sigma}=1.6728$.

On the basis of similar considerations, we choose an exponential model with parameter 0.000004563 for contralateral recurrence for non-carriers. (This is a Weibull model with scale parameter $\sigma=1$; the fitted scale parameter was $\hat{\sigma}=1.02$.) Similarly, we choose a log-normal model with parameters $\hat{\mu}=3.8916$ and $\hat{\sigma}=1.8376$ for distant recurrence for non-mutation carriers. Figures 9 and 10 show the Q-Q plots for the four parametric models; we omit the other graphs for brevity.

(b) Data for mutation carriers are sparse and the plots of the survival probabilities are more helpful than the Q-Q plots in choosing models. Figures 11-13 show these plots for the ipsilateral, contralateral and distant recurrence, respectively, in the case of BRCA1 mutation carriers. In all cases, we choose the log-normal distribution, and the parameters are shown in Table 9.

(c) For women with a BRCA2 mutation, Figures 14 and 15 show plots of survival probabilities contralateral and distant recurrence, respectively, in the case of BRCA2 mutation carriers. Again, we choose the log-normal distribution and the parameters are shown in Table 9. Data were particularly sparse (13 women, 3 contralateral recurrences, 3 distant and none ipsilateral). However, Robson et al. (2005) reported a probability of $80 \%$ of surviving free of contralateral recurrence in a median follow-up 

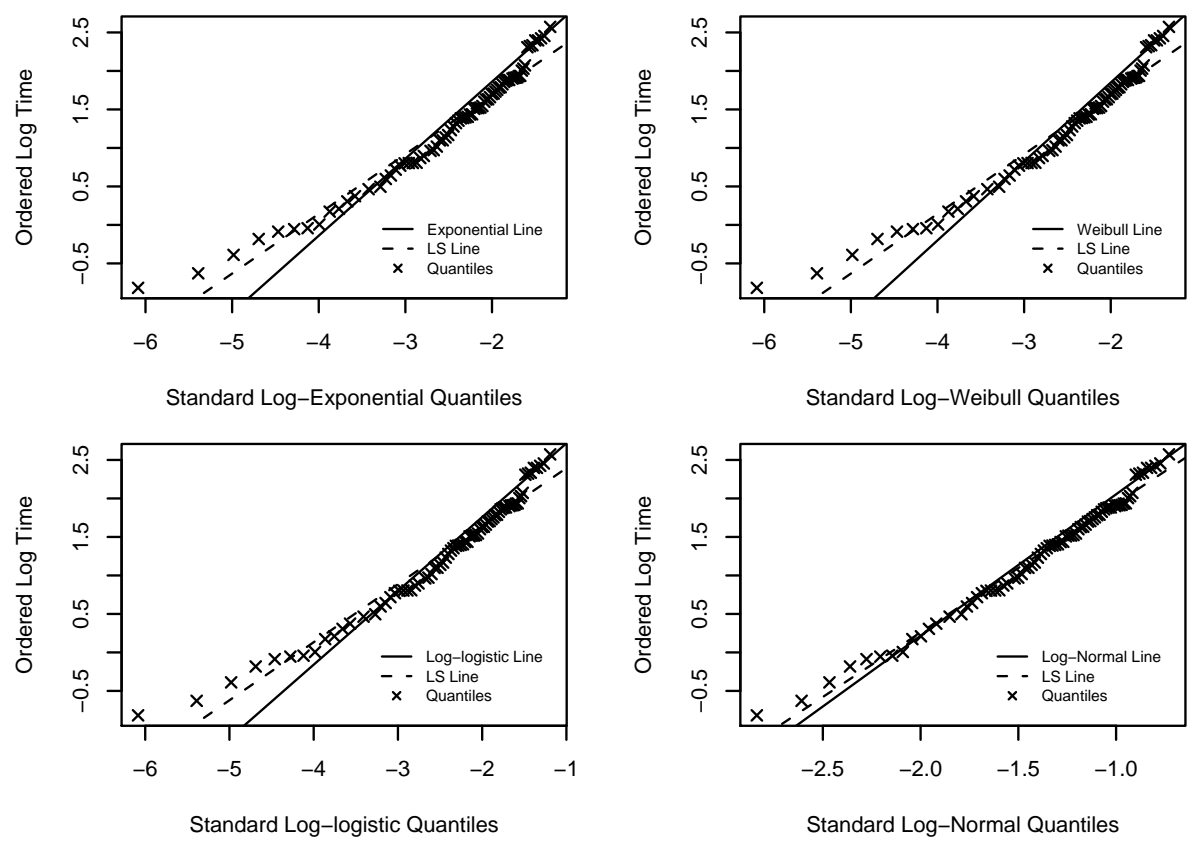

Figure 10: Q-Q plots to test model adequacy of distant recurrence for non-carriers of BRCA1/2 mutations.

Table 9: Log-normal parameter estimates of rates of onset of various types of recurrence for BRCA1/2 mutation carriers.

$\begin{array}{lcclcc}\text { Intensity } & \hat{\mu} & \hat{\sigma} & \text { Intensity } & \hat{\mu} & \hat{\sigma} \\ \mu_{z}^{I R, B C S, B R C A 1} & 4.5214 & 1.9275 & \mu_{z}^{I R, B C S, B R C A 2} & 4.5214 & 1.9275 \\ \mu_{z}^{C R, B C S, B R C A 1} & 3.0337 & 1.6076 & \mu_{z}^{C R, B C S, B R C A 2} & 2.9925 & 1.5962 \\ \mu_{z}^{D R, B C S, B R C A 1} & 2.4373 & 1.6033 & \mu_{z}^{D R, B C S, B R C A 2} & 2.8170 & 1.2797\end{array}$

of 67.4 months, which is quite close to our estimate of $78.6 \%$.

In the absence of any ipsilateral cases, we assume $\mu_{z}^{I R, B C S, B R C A 2}=\mu_{z}^{I R, B C S, B R C A 1}$. There is some support for this from the results of fitting Cox regression models to the risks of contralateral and distant recurrence, with mutation status as a covariate. The hazard ratio (BRCA1/BRCA2) was $1.06(p=0.93)$ for contralateral recurrence and $0.56(p=0.35)$ for distant recurrence, lack of significance in the latter case being also reported by Robson et al. (2005).

\subsection{Recurrence Rates after Primary Treatment of Mastectomy for Locally Advanced Breast} Cancer for Non-carriers

Overgaard et al. (1997) reported a randomized trial with 1,708 women who had undergone mastectomy for locally advanced breast cancer. Of these, 852 women were randomly assigned to receive eight cycles of CMF chemotherapy plus irradiation of the chest wall and regional lymph nodes; the other 856 women received nine cycles of CMF chemotherapy alone. The median length of follow-up was 114 months. The numbers of 
Table 10: Recurrences after mastectomy. Source: Overgaard et al. (1997).

$\begin{array}{lccc} & \text { No. of } & \text { Non-distant } & \text { Distant } \\ \text { Treatment } & \text { Patients } & \text { Recurrence } & \text { Recurrence } \\ \text { (1) Radiotherapy + CMF } & 852 & 119(14 \%) & 287(34 \%) \\ \text { (2) CMF alone } & 856 & 498(58 \%) & 219(26 \%)\end{array}$

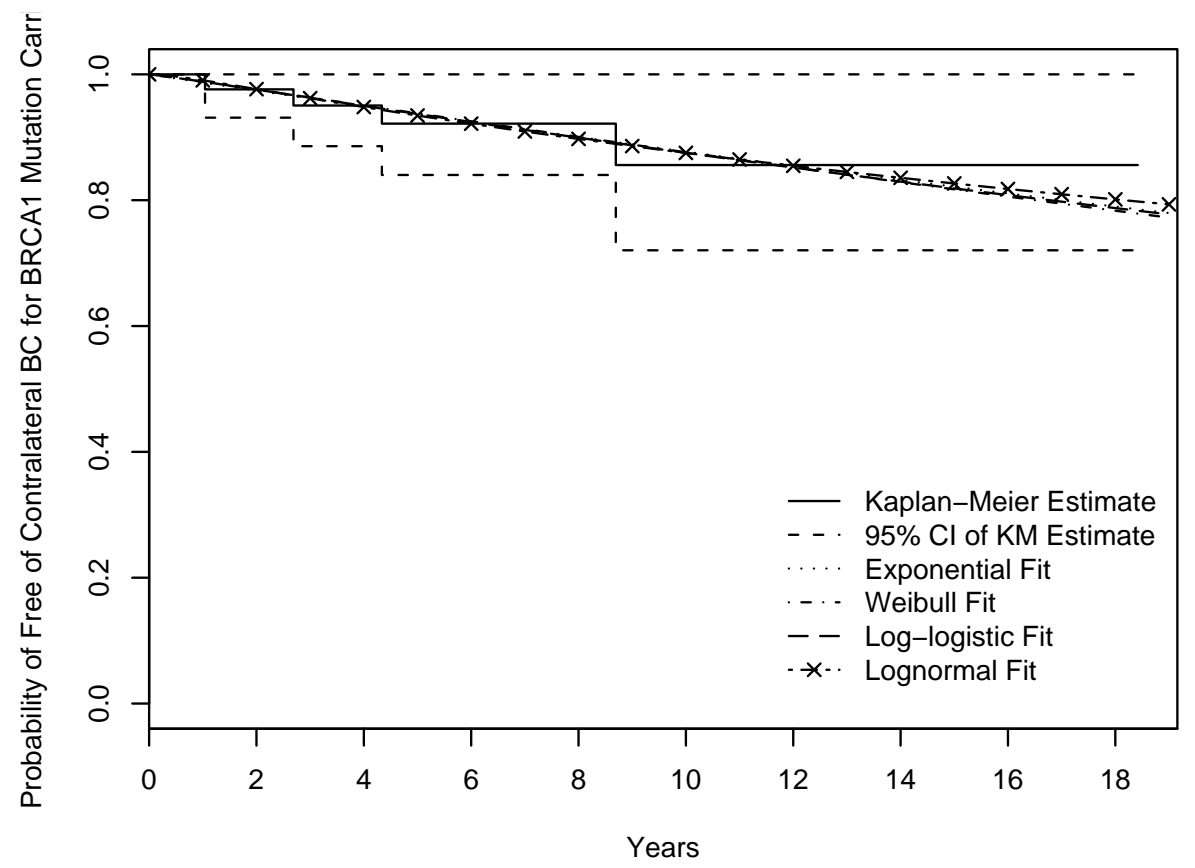

Figure 11: Non-parametric and parametric estimates for probability of free of ipsilateral recurrence for BRCA1 mutation carriers.

non-distant recurrences and distant recurrences are reproduced in Table 10, from which we see that the combination of radiotherapy and chemotherapy after mastectomy reduces the non-distant recurrence substantially compared with chemotherapy alone.

By comparing the results of treatment (1) in Table 10 with the recurrence results of the first 10 years of follow up in Table 7, we see that the risks of non-distant recurrence are similar (14\% and 13.1\%), but the risk of distant recurrence in Table 10 is higher than in Table 7 (34\% and 22.3\%). Other studies also reported similar risks of non-distant recurrence, from 12.5-15\% (Favret et al., 2001; Rustogi et al., 2005). Therefore, we assume the rate of non-distant recurrence is the same as that for early breast cancer after primary treatment of BCS, shown in Section 9.2. In respect of distant recurrence, we assume that the rate of distant recurrence $\mu_{z}^{D R, M A S, N C}$ is a multiple of $\mu_{z}^{D R, B C S, N C}$. Given that the probability of survival free of distant recurrence is $66 \%$ at 114 months, we assume $\mu_{z}^{D R, M A S, N C}=2 \mu_{z}^{D R, B C S, N C}$.

Low et al. (2004) reported a probability of distant recurrence of $53.3 \%(57 / 107)$ in a study of LABC (median follow-up 16.8 years), which is similar to our estimate of $48.2 \%$. 


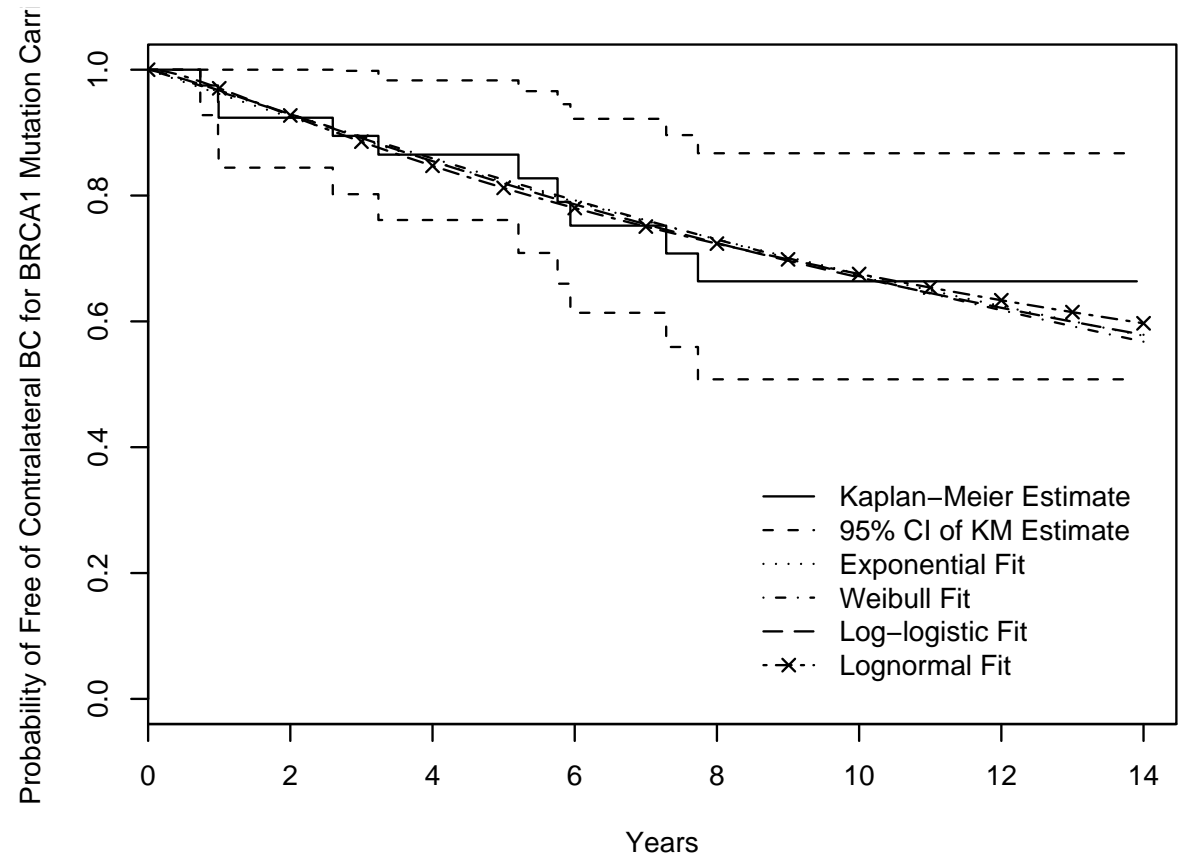

Figure 12: Non-parametric and parametric estimates for probability of free of contralateral recurrence for BRCA1 mutation carriers.

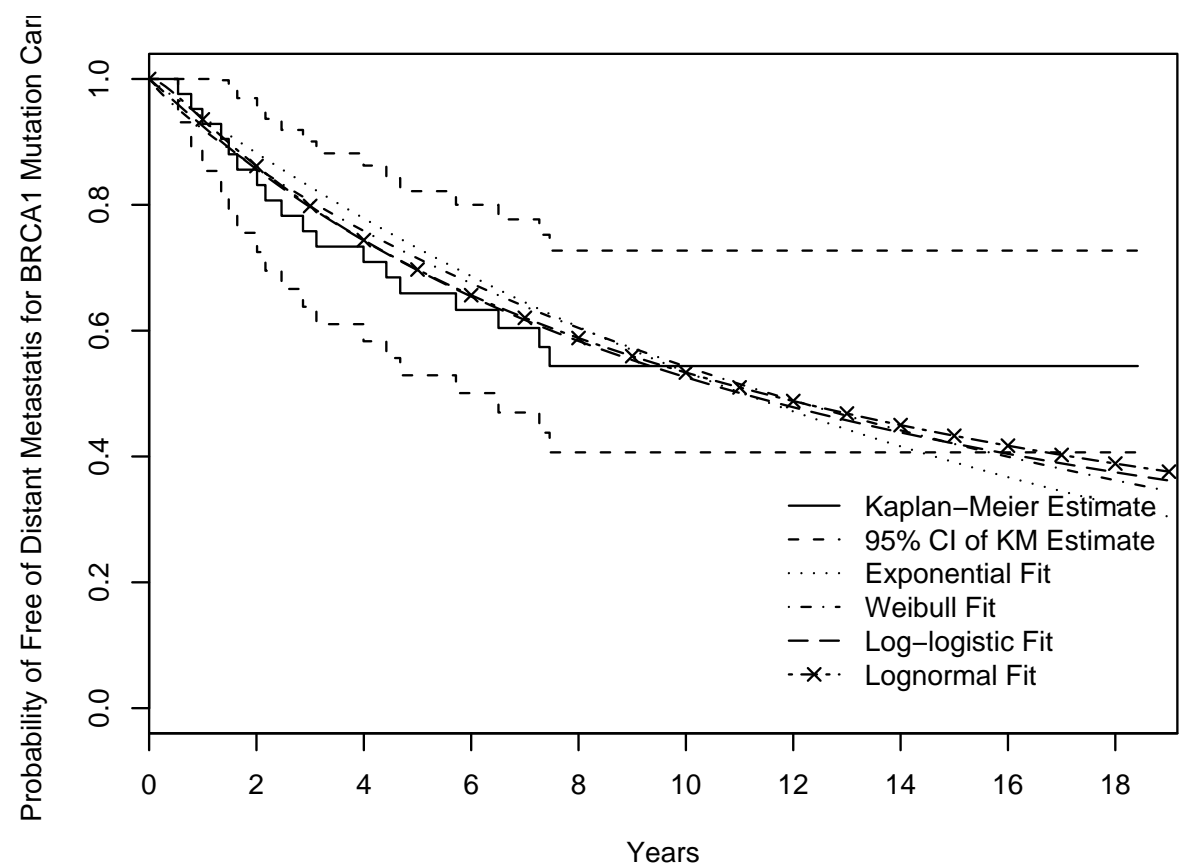

Figure 13: Non-parametric and parametric estimates for probability of free of distant recurrence for BRCA1 mutation carriers. 


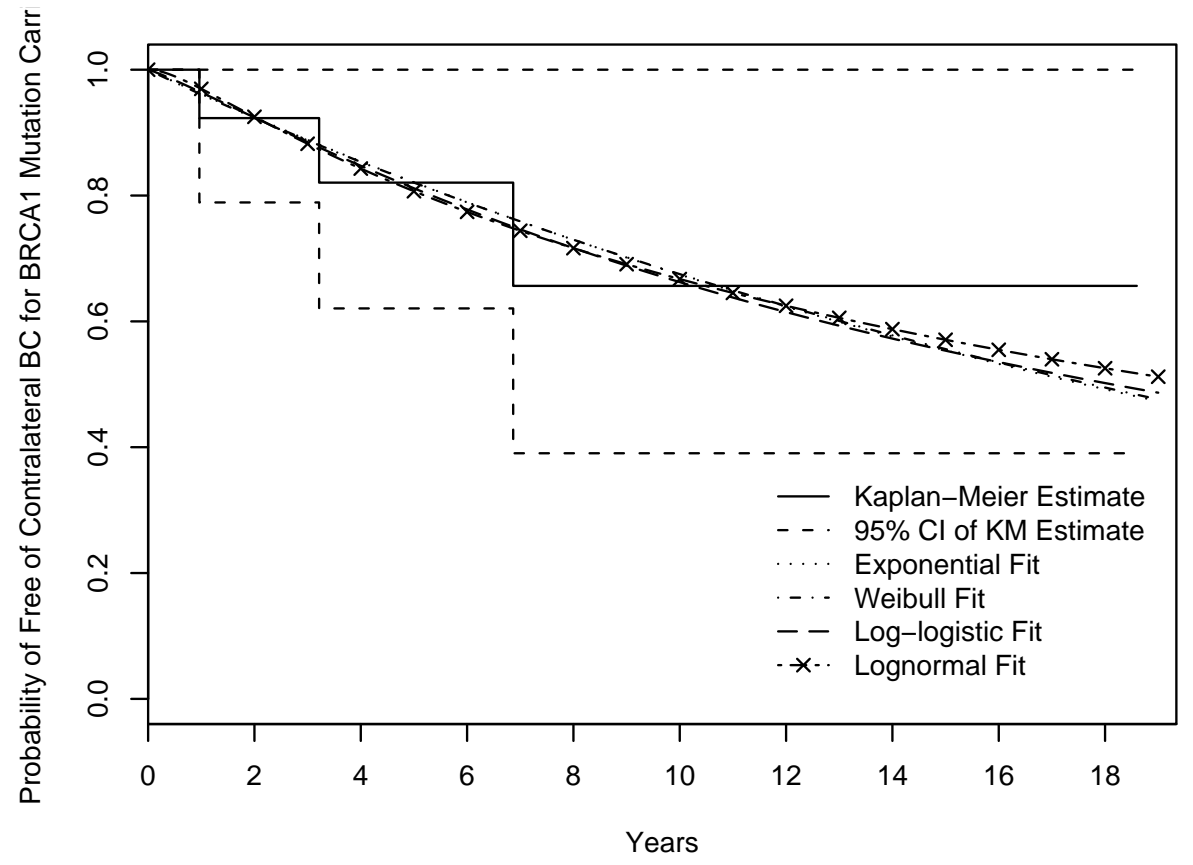

Figure 14: Non-parametric and parametric estimates for probability of free of contralateral recurrence for BRCA2 mutation carriers.

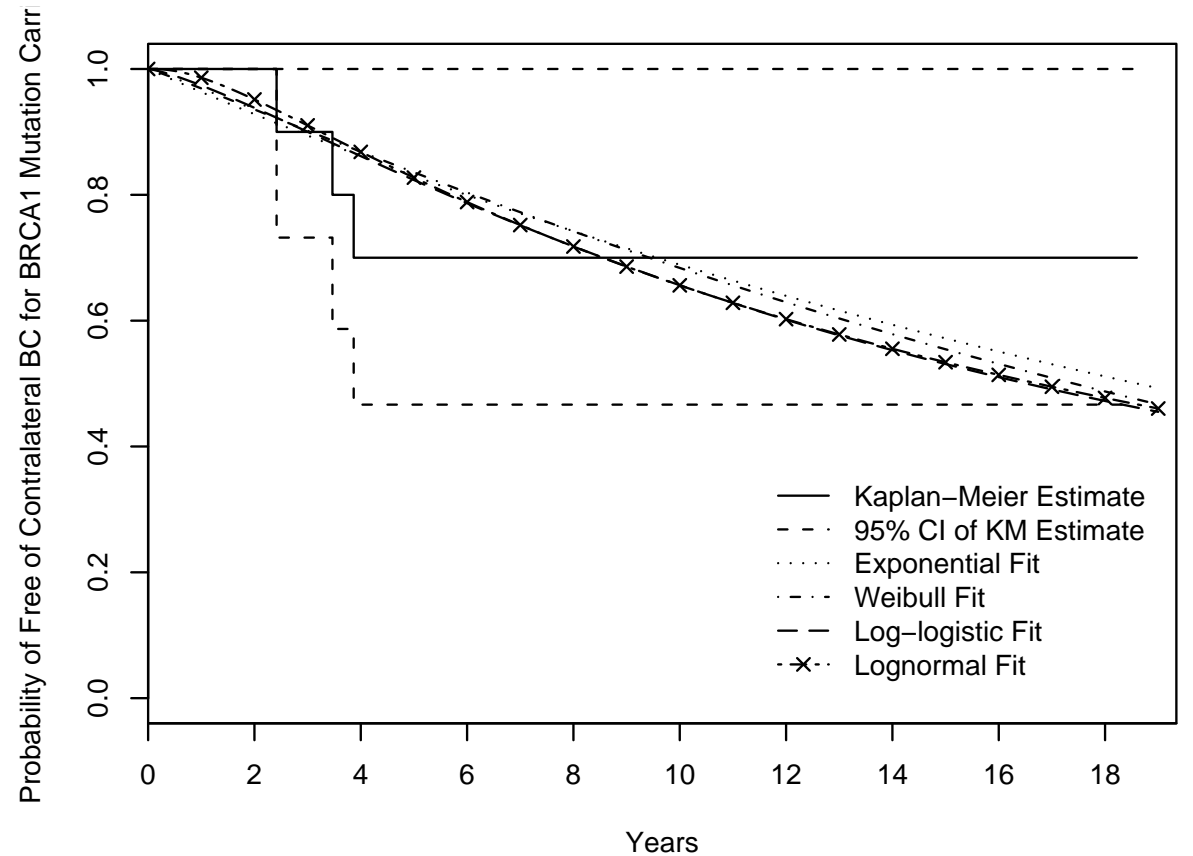

Figure 15: Non-parametric and parametric estimates for probability of free of distant recurrence for BRCA2 mutation carriers. 
Table 11: Recurrences after bilateral mastectomy. Source: Peralta et al. (2000) and Herrinton et al. (2005).

$\begin{array}{lcc} & \text { Peralta } \text { et al. (2000) } & \text { Herrinton et al. (2005) } \\ \text { Number } & 64 & 1072 \\ \text { Median Years of Follow-up } & 6.2 & 5.7 \\ \quad \text { Early BC } & 28(43.8 \%) & 976(91 \%) \\ \text { Locally advanced BC } & 36(56.2 \%) & 96(9 \%) \\ \text { Family History } & 42(65.6 \%) & 375(35 \%) \\ \text { Ipsilateral recurrence } & 10(15.6 \%) & 133(12.4 \%) \\ \text { Contralateral recurrence } & 3(4.7 \%) & 5(0.5 \%) \\ \text { Distant recurrence } & 12(18.8 \%) & 112(10.5 \%)\end{array}$

9.4 Recurrence Rates after Primary Treatment of Bilateral Mastectomy for Locally Advanced Breast Cancer for Mutation Carriers

Given the high rate of contralateral breast cancer for BRCA1/2 mutation carriers, $\mathrm{BM}$ has long been an option for BC patients. The efficacy of $\mathrm{BM}$ in reducing risk for contralateral BC has been confirmed by two studies, Peralta et al. (2000) and Herrinton et al. (2005), which suggest that as well as reducing the incidence of contralateral BC by $90 \%$, BM may also be associated with a significant reduction in BC mortality. Table 11 shows the details of various types of recurrence for women who underwent BM at diagnosis of $\mathrm{BC}$. We observe that the risk of contralateral recurrence is indeed reduced, but the risks of ipsilateral and distant recurrences seem little influenced and were still significantly higher than their counterparts after BCS for non-invasive or early BC. However, we should note that both studies were population-based, and patients with early BC were common in both datasets (or even being in the majority in Herrinton et al. (2005)).

Relevant rates of onset of various recurrences are difficult to estimate directly, since, to our knowledge, randomized trials of the effect of BM for LABC for BRCA1/2 mutation carriers are not available. Along the lines of Section 9.3 we assume that recurrence rates $\mathrm{BM}$ are a constant multiple of recurrence rates after BCS.

Given that no extra risks would be expected after BM compared with BCS, because the volume of breast tissue which contains a mutation was maximally reduced (Turner et al., 1999), the breast cancer type seems a key risk factor. In Section 9.3, we estimated an approximate RR of 2 between LABC and early BC. Based on a Cox model, Robson et al. (2004) reported that the relative risk of positive axillary nodes versus negative nodes in respect of $\mathrm{BC}$ survival is 1.78 (95\% CI, 1.07-2.96; $p=0.03$ ) (positive status of axillary nodes is a key criterion to distinguish LABC). Bonadonna et al. (2005) reported a similar relative risk of 1.67 (95\% CI, $1.28-2.18 ; p=0.0001)$ in respect of recurrence-free survival.

Therefore, we assume a relative risk of 2 , so that $\mu_{z}^{I R, B M, B R C A 1} \approx 2 \times \mu_{z}^{I R, B C S, B R C A 1}$ and similarly for other recurrence types.

\section{Conclusions}

In this Part we parameterised a semi-Markov model of the life history of a woman at risk of $\mathrm{BC}$. Here, we summarise the parts of the model relating to $\mathrm{BC}$ : 
(a) We suppose an individual aged $x$, starting in state 'Normal', could later be detected as having any stage of BC, either by mammographic screening or by clinical methods.

(b) We assume that non-invasive BC can be detected only by screening, while other types of $\mathrm{BC}$ can be detected clinically.

(c) We assume that the patient will receive treatment immediately after the diagnosis of $\mathrm{BC}$. If metastatic BC is diagnosed, the tumour is not curable. If other types of $\mathrm{BC}$ are detected, the patient will undergo surgery plus adjuvant therapies until being able to return to work. In the following years, she would be asked to follow up occasionally in case of tumour recurrence. If non-distant recurrence arises, she will receive treatment until returning to work again; or if distant recurrence is diagnosed, she will die.

(d) If a tumour recurs in an individual with previous experience of non-distant recurrence, we assume that it must be a distant recurrence, because the patient will have undergone mastectomy or BM indicating that most (or all) breast tissues have been excised.

(e) All states have a transition to 'Dead'.

As pointed out by an anonymous referee, the model is capable of extension to model the impact of new adjuvant treatments such as Trastuzumab (better known by its trade name of Herceptin) based on how hormone receptor status affects tumour development. One such preliminary study, though based on a much simpler model, is Macdonald \& Roche (2008).

In Part $\mathrm{V}$ we bring together this model of $\mathrm{BC}$ and a model of IPI business.

\section{ACKNOWLEDGEMENTS}

This work was carried out at the Genetics and Insurance Research Centre at HeriotWatt University, which receives funding from a consortium of insurance companies through the Association of British Insurers. We thank the sponsors for funding, and members of

the Steering Committee for helpful comments at various stages. In particular, we wish to thank Dr. Mark Robson from the Memorial Sloan-Kettering Cancer Center in New York and Dr. William Foulkes and Dr. Pierre Chappuis of McGill University and the Jewish General Hospital in Montreal, for providing the underlying datasets of this study. We thank an anonymous referee for helpful comments on the paper.

\section{REFERENCES}

Antoniou, A.C., Pharoah, P.P.D., Narod, S. et al. (2003). Average risks of breast and ovarian cancer associated with mutations in BRCA1 or BRCA2 detected in case series unselected for family history: A combined analysis of 22 studies. American Journal of Human Genetics, 72, 1117-1130.

Armitage, P. \& Doll, R. (1961). Stochastic models for carcinogenesis. Proceedings of the Fourth Berkeley Symposium on Mathematical Statistics and Probability, 4, 9-38.

Bonadonna, G., Moliterni, A., Zambetti, M. et al. (2005). 30 years' follow up of randomized studies of adjuvant CMF in operable breast cancer: cohort study. British Medical Journal, 330, 217. 
Chen, H.H., Hsieh, H.J. \& Chang, S.H. (2002). Assessing chronic disease progression using non-homogeneous exponential regression Markov models: an illustration using a selective breast cancer screening in Taiwan. Statistics in Medicine, 21, 3369-3382.

Claus,E.B., Risch, N. \& Thompson, W.D. (1991). Genetic analysis of breast cancer in the Cancer and Steroid Hormone study. American Journal of Human Genetics, 48, 232-242.

Claus,E.B., Schildkrant, J.M., Thompson, W.D. \& Risch, N. (1996). The genetic attributable risk of breast and ovarian cancer. Cancer, 77, 2318-2324.

DAY, N.E. \& WALTER, S.D. (1984). Simplified models of screening for chronic disease: estimation procedure from mass screening programmes. Biometrics, 40, 1-14.

Duffy, S.W., TABAr, L., Fagerberg, G. et al. (1991). Breast screening, prognostic factors and survival: Results from the Swedish two county study. British Journal of Cancer, 64, $1133-1138$.

Duffy, S.W., Chen, H.H., Tabar, L. \& Day, N.E. (1995). Estimation of mean sojourn time in breast cancer screening using a Markov chain model of both entry to and exit from the preclinical detectable phase. Statistics in Medicine, 14, 1531-1543.

Duffy, S.W., Day, N.E., TARbar, L. \& Chen, H.H. (1997). Markov models of breast tumour progression: Some age-specific results. Journal of the National Cancer Institute Monographs, 22, 93-97.

Early Breast Cancer Trialists' Collaborative Group (1998). Polychemotherapy for early breast cancer: an overview of the randomized trials. The Lancet, 352, 930-942.

Easton, D.F., Steele, L. et al. (1997). Cancer risks in two large breast cancer families linked to BRCA2 on chromosome 13q12-13. American Journal of Human Genetics, 61, 120-128.

El-Tamer, M., Russo, D. et al. (2004). Survival and recurrence after breast cancer in BRCA1/2 mutation carriers. Annals of Surgical Oncology, 11, 157-164.

Fagerberg, C.J.G., Baldetorp, L., Gröntoft, O. et al. (1985). Dilemmas in locally advanced breast disease. The Breast Journal, 7, 131-137.

Favret, A.M., Carlson, R.W., Goffinet, D.R. et al. (2001). Effects of repeated mammographic screening on breast cancer stage distribution. Acta Radiology (Oncology), 24, 465.

Fisher, B., Anderson, S., Bryant, J. et al (2002). Twenty-year follow-up of a randomized trial comparing total mastectomy, lumpectomy and lumpectomy plus irradiation for the treatment of invasive breast cancer. New England Journal of Medicine, 347, 1233-1241.

Ford, D., Easton, D.F., Bishop, D.T., Narod, S.A. \& Goldgar, D.E. (1994). Risks of cancer in BRCA1 mutation carriers. The Lancet, 343, 692-695.

Foulkes, W.D., Wong, N., Brunet, J.S. et al. (1997). Germ-line BRCA1 mutation is an adverse prognostic factor in Ashkenazi Jewish women with breast cancer. Clinical Cancer Research, 3, 2465-2469.

Futreal, P.A., Liu, Q., Shattuck-Eldens, D., Cochran, C., Harshman, K., Tavtigian, S., Bennett, L.M., Haugen-Strano, A., Swensen, J. \& Miki, Y. (1994). BRCA1 mutations in primary breast and ovarian carcinomas. Science, 266, 120-122.

Gui, E.H., Lu, B., Macdonald, A.S., Waters, H.R. \& Wekwete, C.T. (2006). The genetics of breast and ovarian cancer III: A new model of family history with insurance application. 2006, 338-367, 
Haffy, B.G., Harrold, E. et al. (2002). Outcome of conservatively managed early onset breast cancer by BRCA1/2 status. The Lancet, 359, 1471-1477.

Haites, N. And Gregory, H. (2002). Familial breast and ovarian cancer: genetics, screening and management. Part 2: Overview of the clinical genetics of breast cancer. Cambridge University Press.

Hartmann, L.C., Sellers, T.A. et al. (2001). Efficacy of bilateral prophylactic mastectomy in BRCA1 and BRCA2 mutation carriers. Journal of the National Cancer Institute, 93, $1633-1637$.

Herrinton, L.J., Barlow, W.E., Yu, O. et al. (2005). Efficacy of prophylactic mastectomy in women with unilateral breast cancer: A cancer research network project. Journal of the National Cancer Institute, 23, 4251-4253.

Low, J.A., Berman, A.W., Steinberg, S.M. et al. (2004). Long-term follow-up for locally advanced and inflammatory breast cancer patients treated with multimodality therapy. Journal of Clinical Oncology, 22, 4067-4074.

Macdonald, A.S. \& Roche, E. (2008). How will Trastuzumab affect life insurance?. Genetics and Insurance Research Center Report 08/1, Heriot-Watt University, Edinburgh.

Macdonald, A.S., Waters, H.R. \& Wekwete, C.T. (2003a). The genetics of breast and ovarian cancer I: A model of family history. Scandinavian Actuarial Journal, 2003, 1-27.

Macdonald, A.S., Waters, H.R. \& Wekwete, C.T. (2003b). The genetics of breast and ovarian cancer II: A model of critical illness insurance. Scandinavian Actuarial Journal, 2003, 28-50.

Meijers-Heijboer, H., Verhoog, L.C. et al. (2000). Presymptomatic DNA testing and prophylactic surgery in families with a BRCA1 or BRCA2 mutation. The Lancet, $\mathbf{3 5 5}$, 2015-2020.

Meijers-Heijboer, H., van Geel, B. et al. (2001). Breast cancer after prophylactic bilateral mastectomy in women with a BRCA1 or BRCA2 mutation. New England Journal of Medicine, 345, 159-164.

Miki, Y., Swensen, J., Shattuck-Eidens, D. et al. (1994). A strong candidate for the breast and ovarian cancer susceptibility gene BRCA1. Science, 266, 61-71.

NiH Consensus Development Panel (2000). Adjuvant therapy for breast cancer: NIH Consensus Statement 2000 November 1-3. cited 2006 April 4, 17(4), 1-23.

Nicoletto, M.O., Donach, M. et al. (2001). BRCA-1 and BRCA-2 mutations as prognostic factors in clinical practice and genetic counseling. Cancer Treatment Reviews, 27, 295-304.

O.N.S. (1999). Cancer 1971-1997 CD-ROM. Office for National Statistics, London.

Overganrd, M., Hansen, P.S., Overganrd, J. et al. (1997). Postoperative therapy in high risk premenopausal women with breast cancer who receive adjuvant chemotherapy. Danish Breast Cancer Cooperative Group 82b trial. New England Journal of Medicine, 337, 949-955.

Peralta, E.A., Ellenhorn, J.D., Wagman, L.D. et al. (2000). Contralateral prophylactic mastectomy improves the outcome of selected patients undergoing mastectomy for breast cancer. American Journal of Surgery, 180, 439-445.

Pierce, L.J., Strawderman, M., Narod, S.A. et al. (2000). Effect of radiotherapy after breast-conserving treatment in women with breast cancer and germline BRCA1/2 mutations. Journal of Clinical Oncology, 18, 3360-3369. 
Rustogi, A., Budrukkar, A., Dinshaw, K., Jalali, R. (2005). Management of locally advanced breast cancer: Evolution and current practice. Journal of Cancer Research and Therapy, 1, 21-30.

Robson, M.E., Chappuis, P.O., Satagopan, J. et al. (2004). A combined analysis of outcome following breast cancer: differences in survival based on BRCA1/BRCA2 mutation status and administration of adjuvant treatment. Breast Cancer Research, 6, R8-R17.

Robson, M.E., Svahn, T., McCormick, B. et al. (2005). Appropriateness of breastconserving treatment of breast carcinoma in women with germline mutations in BRCA1 or BRCA2: A clinic-based Series. Cancer, 103, 44-51.

Schwartz, M.D., Lerman, C., Brogan, B. et al. (2004). Impact of BRCA1/BRCA2 counseling and testing on newly diagnosed breast cancer patients. Journal of Clinical Oncology, 22, 1823-1829.

Scott, C.L., Jenkins, M.A., Southey, M.C. et al. (2003). Average age-specific cumulative risk of breast cancer according to type and site of germline mutations in BRCA1 and BRCA2 estimated from multiple-case breast cancer families attending Australian family cancer clinics. Human Genetics, 112(5-6), 542-551.

SEIfFERT, J.E. (EDITOR) (1993). SEER program: comparative staging guide for cancer. National Cancer Institute, NIH Pub. No. 93-3640, 1993.

Steering Committee on Clinical Practice Guidelines for the Care and Treatment OF Breast CANCER (1998). Clinical practice guidelines for the care and treatment of breast cancer. CMAJ, 158(Suppl 3), S71-81.

Steering Committee on Clinical Practice Guidelines for the Care and Treatment OF BREAST CANCER (2001). Questions and answers on breast cancer: a guide for women and their physicians. Canadian Medical Association, available electronically at www.cma.ca.

Tabar, L., Fagerberg, G., Chen, H.H., Duffy, S.W., Gad, A. (1996). Tumour development, history and grade of breast cancers prognosis and progression. International Journal of Cancer, 66, 413-419.

Rebbeck, T., Friebel, T. et al. (2004). Bilateral prophylactic mastectomy reduces breast cancer risk in BRCA1 and BRCA2 mutation carriers: The PROSE Study Group. Journal of Clinical Oncology, 22, 1055-1062.

Tabar, L., Fagerberg, C.J.G., Gad, A. et al. (1985). Reduction in mortality from breast cancer after mass screening with mammography. The Lancet, 325, 829-832.

Tabar, L., Duffy, S.W. \& Krusemo, U.B. (1987). Detection method, tumour size and node metastases in breast cancers diagnosed during a trial of breast cancer screening. European Journal of Cancer, 23, 959.

The Breast Cancer Linkage Consortium (1999). Cancer risks in BRCA2 mutation carriers. Journal of the National Cancer Institute, 91, 1310-1316.

Turner, B.C., Harrold, E., Matloff, E. et al. (1999). BRCA1/BRCA2 germline mutations in locally recurrent breast cancer patients after lumpectomy and radiation therapy: implications for breast-conserving management in patents with BRCA1/BRCA2 mutations. Journal of Clinical Oncology, 17, 3017-3024.

Verhoog, L.C., Brekelmans, C.T.M. et al. (1998). Survival and tumour characteristics of breast-cancer patients with germline mutations of BRCA1. The Lancet, 351, 316-321. 
Verhoog, L.C., Brekelmans, C.T.M. et al. (1999). Survival and tumour characteristics of breast-cancer patients with germline mutations of BRCA2. Journal of Clinical Oncology, 17, 3396-3402.

Veronesi, U., Banfi, A., SACCOzzi, R. et al (1977). Conservative treatment of breast cancer: a trial in progress at the Cancer Institute in Milan. Cancer, 39 Suppl, 2822-2826.

Veronesi, U., Saccozzi, R., Del Vecchio, M. et al (1981). Comparing radical mastectomy with quadrantectomy, axillary dissection, and radiotherapy in patients with small cancers of the breast. New England Journal of Medicine, 305, 6-11.

Veronesi, U., Cascinelli, N. et al (2002). Twenty-year follow-up of a randomized study comparing breast-conserving surgery with radical mastectomy for early breast cancer. New England Journal of Medicine, 347, 1227-1232.

Veronesi, U., Boyle, P., Goldhirsch, A., Orecchia, R., Viale, G. (2005). Breast Cancer. The Lancet, 365, 1727-1741.

Wooster, R., Bignell, G., Lancaster, J., Swift, S., Seal, S., Mangion, J., Collins, N., Gregory, S., Gumbs, C. \& Micklem, G. (1995). Identification of the breast cancer susceptibility gene BRCA2. Nature, 378, 789-792.

Young, J.L. Jr., Roffers, S.D., Ries, L.A.G., Fritz, A.G., Hurlbut, A.A. (editors) (2001). SEER summary staging manual-2000: codes and coding instructions. National Cancer Institute, NIH Pub. No. 01-4969, Bethesda, MD, 2001. 